\title{
Measuring water level in rivers and lakes from lightweight Unmanned Aerial Vehicles
}

Bandini, Filippo; Jakobsen, Jakob; Olesen, Daniel Haugård; Reyna-Gutiérrez, José Antonio; BauerGottwein, Peter

Published in:

Journal of Hydrology

Link to article, DOI:

10.1016/j.jhydrol.2017.02.038

Publication date:

2017

Document Version

Peer reviewed version

Link back to DTU Orbit

Citation (APA):

Bandini, F., Jakobsen, J., Olesen, D. H., Reyna-Gutiérrez, J. A., \& Bauer-Gottwein, P. (2017). Measuring water level in rivers and lakes from lightweight Unmanned Aerial Vehicles. Journal of Hydrology, 548, 237-250.

https://doi.org/10.1016/j.jhydrol.2017.02.038

\section{General rights}

Copyright and moral rights for the publications made accessible in the public portal are retained by the authors and/or other copyright owners and it is a condition of accessing publications that users recognise and abide by the legal requirements associated with these rights.

- Users may download and print one copy of any publication from the public portal for the purpose of private study or research.

- You may not further distribute the material or use it for any profit-making activity or commercial gain

- You may freely distribute the URL identifying the publication in the public portal 


\section{Measuring water level in rivers and lakes from}

\section{lightweight Unmanned Aerial Vehicles}

*Corresponding author: $\underline{\text { bban@env.dtu.dk }}$

${ }^{1}$ Department of Environmental Engineering, Technical University of Denmark, 2800, Lyngby, Denmark

\section{Highlights}

12 -Water level of rivers and lakes can be measured by Unmanned Aerial Vehicles.

13 -Unmanned Aerial Vehicles ensure high accuracy and spatial resolution.

14 -The measuring system consists of a ranging sensor and a GNSS receiver.

15 -Among the ranging sensors, the radar has the highest accuracy and longest range.

16 The camera-laser sensor is preferred for narrow field of view to water surface.

17

18 Abstract 
19 The assessment of hydrologic dynamics in rivers, lakes, reservoirs and wetlands requires

20 measurements of water level, its temporal and spatial derivatives, and the extent and dynamics of

21 open water surfaces. Motivated by the declining number of ground-based measurement stations,

22 research efforts have been devoted to the retrieval of these hydraulic properties from spaceborne

23 platforms in the past few decades. However, due to coarse spatial and temporal resolutions,

24 spaceborne missions have several limitations when assessing the water level of terrestrial surface

25 water bodies and determining complex water dynamics. Unmanned Aerial Vehicles (UAVs) can

26 fill the gap between spaceborne and ground-based observations, and provide high spatial

27 resolution and dense temporal coverage data, in quick turn-around time, using flexible payload

28 design. This study focused on categorizing and testing sensors, which comply with the weight

29 constraint of small UAVs (around $1.5 \mathrm{~kg}$ ), capable of measuring the range to water surface.

30 Subtracting the measured range from the vertical position retrieved by the onboard Global

31 Navigation Satellite System (GNSS) receiver, we can determine the water level (orthometric

32 height). Three different ranging payloads, which consisted of a radar, a sonar and an in-house

33 developed camera-based laser distance sensor (CLDS), have been evaluated in terms of

34 accuracy, precision, maximum ranging distance and beam divergence. After numerous flights,

35 the relative accuracy of the overall system was estimated. A ranging accuracy better than $0.5 \%$

36 of the range and a maximum ranging distance of $60 \mathrm{~m}$ were achieved with the radar. The CLDS

37 showed the lowest beam divergence, which is required to avoid contamination of the signal from

38 interfering surroundings for narrow fields of view. With the GNSS system delivering a relative

39 vertical accuracy better than $3-5 \mathrm{~cm}$, water level can be retrieved with an overall accuracy better

40 than $5-7 \mathrm{~cm}$.

41 
42 Keywords: UAV; water level; radar; sonar; laser; GPS;

\section{1. Introduction}

44 Extreme hydro-climatic events such as droughts, floods and heavy precipitation have increased

45 the awareness that knowledge of spatial and temporal variation of open water surfaces is

46 important (Alsdorf et al., 2007). In order to achieve a better quantitative understanding of

47 hydrologic processes and to increase sharpness and reliability of hydrologic predictions,

48 observations of hydrological variables, such as surface water area, water level (h), its slope

$49(\partial \mathrm{h} / \partial \mathrm{x})$ and its temporal change $(\partial \mathrm{h} / \partial \mathrm{t})$ are required. However, ground-based measurements of

50 terrestrial water bodies are limited to networks of measuring stations. In-situ stations provide

51 point observations that are often spaced too far apart to capture spatial patterns. Often, in-situ

52 observation technology fails during extreme events. Furthermore, globally, the availability of in-

53 situ hydrologic observation stations has been declining in the recent past (Lawford et al., 2013).

54 Hence, remote sensing datasets have become increasingly popular in hydrology. Remote sensing

55 techniques are presently unable to observe river discharge directly, however spatial and temporal

56 variation of water level has been routinely observed using spaceborne or airborne platforms.

57 Although most satellite altimetry missions were not designed primarily for monitoring

58 continental waters, water levels of continental water surfaces retrieved by Seasat,

59 TOPEX/Poseidon, Jason-1 and 2, GFO, ERS 1 and 2, ENVISAT have a measurement accuracy

60 that is well understood and generally on the order of a few tens of centimeters (Calmant et al.,

61 2008). This accuracy can be improved for larger lakes and rivers by averaging over large water

62 surfaces (Birkett, 1998; Birkett et al., 2002; Frappart et al., 2006). The satellite CryoSat-2 carries

63 a Synthetic Aperture Interferometric Radar Altimeter (SIRAL) which is a new generation radar

64 altimeter (Wingham et al., 2006) with a spatial resolution of around $300 \mathrm{~m}$ (Villadsen et al., 
65 2015). When operating in SARIn mode, a correction of the cross-track slope can be performed

66 and waveform analysis allows separation between water and surrounding topography

67 (Kleinherenbrink et al., 2014) resulting in an accuracy of the retrieved water level of just a few

68 decimeters (Kleinherenbrink et al., 2015). Spaceborne LIDARs such as the Geoscience Laser

69 Altimeter System (GLAS) have been shown to provide water level measurements with higher

70 accuracy than radar altimeters such as TOPEX/Poseidon (Zhang and Xie, 2010). Still, GLAS

71 has a ground footprint that is around $65 \mathrm{~m}$ (Schutz et al., 2005) and retrieves observations at

72 irregular temporal intervals. Therefore, the main limitations of conventional satellite radar and

73 laser altimetry are low spatial resolution, local coverage (for short repeat orbit missions) and low

74 temporal resolution (for long repeat missions such as CryoSat). In order to overcome these

75 limitations, the forthcoming Surface Water and Ocean Topography (SWOT) satellite mission

76 will build on the heritage of the imaging interferometric radars such as the Shuttle Radar

77 Topography Mission (SRTM) (Kiel et al., 2006; LeFavour and Alsdorf, 2005; Rodriguez et al.,

78 2006). However, spaceborne sensors will always face problems of: i) large ground footprints,

79 which result in relatively low spatial resolution; ii) fixed orbit configurations, which may be

80 inappropriate for high-resolution coverage of local water bodies; iii) coarse temporal resolution

81 and/or the non-regular revisit intervals. These limitations restrict their ability to measure the

82 temporal and spatial variation of the water level with the accuracy needed for determining the

83 hydraulics of complex rivers and flood waves.

84 Airborne LIDAR techniques have the advantages of better tracking of terrestrial water bodies,

85 improved spatial resolution, clear segmentation between land and water surfaces and a higher

86 accuracy (Schumann et al., 2008). However, airborne LIDAR surveys are expensive and their

87 success depends on surveying conditions (e.g. topography and geometry, vegetation cover, size 
88 of the water body). For this reason, digital elevation models and digital surface models retrieved

89 by airborne LIDAR are not universally available and are normally not retrieved during periods of

90 hydrological interest such as flood events.

91 UAVs (Unmanned Aerial Vehicles) and in particular micro-UAVs (payload less than 1.5-2 kg),

92 represent the latest frontier in land and water monitoring because of low-altitude flight, low cost

93 and flexible payload design (Anderson and Gaston, 2013). In recent years, miniaturized

94 components (GNSS receivers, inertial measurement units, autopilots) have advanced (Watts et

95 al., 2012), and UAVs have been used also for a wide range of hydrological applications such as

96 fluvial monitoring; river bathymetry and photogrammetric DEM generation using very high

97 resolution (VHR) imagery (Lejot et al., 2007); water velocity measurements using large-scale

98 particle image velocimetry (LSPIV) (Detert and Weitbrecht, 2015; Tauro et al., 2016, 2015).

99 Moreover, UAVs have attracted great interest for monitoring of environmental disasters and

100 floods (Luo et al., 2015). UAVs are low-cost platforms that have unique capabilities to access

101 hostile or inaccessible environments that need to be urgently monitored. Moreover, they ensure

102 tracking of water surfaces better than satellite technology. However, for LIDAR and SAR

103 systems, the tradeoff between performance, cost and size/weight is still a challenge to be solved

104 before their application in UAV remote sensing (Colomina and Molina, 2014).

105 In this paper, we demonstrate the possibility to acquire measurements of water level by a ranging 106 system that includes a ranging sensor (radar, CLDS or sonar) and a GNSS receiver. The ranging

107 technology described in this paper provides water level measurements with higher accuracy than

108 spaceborne or airborne altimetry. Moreover, it ensures a spatial resolution ideal for measuring

109 the two dimensional spatial variability of small rivers and their interaction with floodplains (Lee

110 et al., 2011). Lastly, the newly developed CLDS can acquire ranges to water surfaces when only 
111 narrow fields of view are available. The CLDS is specifically developed for applications in

112 vegetated environments or inside sinkholes in karst environments.

\section{2. Materials and Methods}

\subsection{General concept}

115 To acquire accurate water level (height above mean sea level) of open water surfaces, the UAV

116 must be equipped with: i) accurate lightweight sensors for measuring the range to water surface

117 ii) a high accuracy dual frequency GNSS receiver and antenna. Installation of an in-situ dual

118 frequency GNSS master station is needed for differential corrections. The general concept is

119 illustrated in Fig. 1.

120

121

\section{Fig. 1.}

124 The ellipsoidal height of the water surface is measured by subtracting the range measured by a

125 ranging sensor from the vertical position retrieved by the onboard GNSS receiver. Afterwards

126 the orthometric height can be retrieved from the ellipsoidal height if the geoid height is known

127 (Featherstone, 2001). For the purpose of this work, a hexacopter has been assembled from

128 TAROT-RC components and has been equipped with DJI Naza-M2 flight controller. The

129 hexacopter is able to fly at least 12 minutes carrying a payload of at most $2 \mathrm{~kg}$. The choice of the

130 ranging sensors was constrained by: i) maximum weight of the payload, ii) a reasonable price

131 necessary for flexible operations, iii) sensor interfaces that allow time synchronization with the

132 GNSS receiver through a microprocessor. The selected ranging sensors included two off-the- 
133 shelf sensors (a radar, a sonar) and the in-house developed CLDS. The total cost of the platform

134 is ca. 7000 euros. This cost includes the drone, the onboard GNSS system, the inertial

135 measurement unit (IMU), the three tested sensors and the microprocessor unit.

136 Fig. 2 shows the arrangement of the drone payload.

145 The radar is the ARS 30X model developed by Continental as anti-collision system for the 146 automotive industry (market price: $3200 \mathrm{EUR}$ ). It weighs around $350 \mathrm{~g}$ and consists of a $77 \mathrm{GHz}$

147 radar sensor with a mechanical scanning antenna. It measures the range to targets using FMCW

148 (Frequency Modulated Continuous Wave) with a sampling frequency of 15 measurements per

149 second. It provides up to 32 targets in near range and up to 64 targets in far range with a 150 resolution of $0.10 \mathrm{~m}$. Each individual target angle is provided with a resolution of $0.1^{\circ}$.

152 The sonar is the MB7386 model from MaxBotix (market price: 150 EUR). It weighs around 50g 153 and consists of a $42 \mathrm{kHz}$ ultrasonic sensor (6 Hz reading rate) with internal temperature 
154 compensation, noise tolerance and clutter rejection. Its maximum ranging capability is up to 10

$155 \mathrm{~m}$.

\subsubsection{Camera-based laser distance sensor (CLDS)}

157 This ranging sensor is a laser camera-based solution recently developed at Technical University 158 of Denmark (Reyna Gutierrez, 2013). It weighs around $350 \mathrm{~g}$. It was inspired by the measuring 159 procedure proposed by Danko (2004). The range distance to the target is estimated by measuring 160 the angle at which laser light enters the camera. The original methodology is expanded in this 161 work to include corrections for tilting and rotation angles of the aircraft. An efficient automatic 162 algorithm for identifying the laser dots on the water surface was developed. Our prototype 163 consists of two laser pointers (100 $\mathrm{mW}$ laser diodes) and a complementary metal-oxide-

164 semiconductor (CMOS) camera. The camera resolution is 20.2 megapixels. The camera is 165 triggered by the on-board single board computer (SBC) with an image rate of 1 frame every 2.5

166 seconds. The total manufacturing cost of this CLDS system is around 800 EUR. The current

167 design of the distance-meter includes a digital camera mounted at the center between the two 168 laser pointers. Fig. 3 shows the geometrical configuration of the camera. Range to water surface 169 is measured by illuminating the water surface with the laser pointers and taking a picture of the 170 illuminated water surface. When light emitted by laser pointers hits the water surface, bright dots

171 are formed at the interface between water and air. Due to scattering processes (in particular 172 Rayleigh and Mie scattering), some portion of the radiation is reflected in the direction of the 173 camera and an estimation of the range to water surface is possible. 
176 The angle $\alpha$ is a design parameter. The CLDS was built with $\alpha=90^{\circ}$ to simplify the measuring 177 concept and the derivation of the formulas. The CLDS shown in Fig. 3 is exactly symmetrical.

178 Indeed, only one laser would be sufficient to acquire the range to the surface; nevertheless, two

179 laser pointers improve error assessment and system accuracy.

180 The value of the measured range Hm can be computed by measuring the angle $\theta^{\prime}$ at which light

181 enters the camera, i.e. from equation (1).

$$
H m=\frac{A}{\tan \theta^{\prime}}
$$

182

183 Alternatively, the measured range $\mathrm{Hm}$ can be obtained through equation (2)

$$
H m=\frac{A \cdot f}{\operatorname{ImD}}
$$

184 Where ImD (Image distance) is the distance between the center of the image and the recorded 185 light source. A calibration procedure is needed to convert from the number of pixels from the 186 center of the image (PFC) to $\mathrm{ImD}$ as shown in equation (3)

$$
\operatorname{ImD}=P F C \cdot d_{p p 1}+d_{0}
$$

187 Where $\mathrm{d}_{\mathrm{pp} 1}$ and $\mathrm{d}_{0}$ are the coefficients of the first-order polynomial producing the best least188 squares fit to the data. Equations (2) and (3) can be applied only when the focal length (f) of the 189 camera is exactly and the focus is constantly set to infinity. Otherwise, the calibration procedure 190 needs to estimate the angle $\theta^{\prime}$ directly from the number of pixels (PFC) as shown in equation (4).

$$
\theta^{\prime}=\mathrm{PFC} \cdot r_{p p 1}+r_{0}
$$


191 Where $r_{p p 1}$ and $r_{0}$ are the coefficients of the first-order polynomial producing the best least-

192 squares fit to the data. The calibration procedure, which has to be performed to estimate the $r_{p p 1}$

193 and $r_{0}$ coefficients, is presented in the appendix. The calibration procedure allows estimation of

194 the angle $\theta^{\prime}$ by measuring PFC, without having to consider the linear or nonlinear intrinsic

195 camera parameters, such as focal length and lens distortion.

196 Onboard the UAV, tilting and rotation cause a displacement of the light sources from their

197 equilibrium position. The changes in the geometrical relationships generate an error in the

198 estimation of the true range distance (hereafter defined as $\mathrm{Ht}$ ) between the sensor of the camera

199 and the water surface. Tilting is the angle between the plane on which the camera and laser are

200 located, i.e. the axis of the CLDS, and the horizontal plane (angle $\beta$ as shown in Fig. 4). Rotation

201 occurs between the vertical line and the optical axis of the camera (angle $\delta$ as shown in Fig. 5).

Fig. 4

Fig. 5

202 If tilting pushes the light source below the axis of the distance meter, formula (5) can be used to 203 obtain the true range $(\mathrm{Ht})$ between the camera and the water surface: 


$$
\mathrm{Ht}=[(H m+A \cdot \tan \beta) \cos \beta] \cdot \cos \delta
$$

204 Conversely, if the tilting pushes the light source above the axis of the CLDS, formula (6) can be 205 used:

$$
\mathrm{Ht}=[(H m-A \cdot \tan \beta) \cos \beta] \cdot \cos \delta
$$

206 If pitch and roll angles are retrieved on board the UAV, the measured range can be corrected

207 according to equation (5) and (6) (Reyna Gutierrez, 2013). If the angles are not retrieved on

208 board, the resulting error on the range can be estimated as shown in Fig. 6. Numerous tests have

209 been conducted in order to determine the best configuration of the CLDS in terms of: i) arm

210 length A, ii) wavelengths of the two laser pointers, iii) optimal camera configuration parameters

211 such as optical zoom and resolution.

212 The arm length choice affects the measuring range function, as shown in Fig. 7.

Fig. 6

Fig. 7

213 Fig. 7 shows that the resolution of the measurements depends on the derivative of the range

214 function. Hence, a longer arm will result in higher resolution, especially for longer ranges.

215 Indeed, in Fig. 7, the smoothest curve is for an arm length of $0.6 \mathrm{~m}$. However, the payload size of

216 small UAVs is limited and thus a $30 \mathrm{~cm}$ arm was chosen for our tests. The wavelengths of the

217 two laser pointers were chosen as $450 \mathrm{~nm}$ and $531 \mathrm{~nm}$, because reflectivity of water is relatively 
218 high at these wavelengths as a consequence of the optical proprieties of water as described in

219 Hale and Querry (1973).

220 When the laser light hits the water surface, a bright dot is formed at the point of contact.

221 However, additional bright spots might be visible due to reflection from the riverbed and due to

222 additional scattering processes caused by water waves. To identify the two dots formed by laser

223 reflection, an automatic identification algorithm was developed consisting of the following

224 computational steps: i) the RGB image is converted to Hue, Saturation and Value (HSV) image.

225 Quasi-circular shapes in the image are found through circular Hough transform (Yuen et al.,

226 1990). In case there are multiple circles in the image, the two circles (one generated by the left

227 laser and one by the right laser) with the highest mean Value (V) are considered to be the contact

228 spots. Thereafter, ii) the brightest pixel (pixel with the highest Value) is identified inside each of

229 the two circles (laser dots). The brightest pixel typically lies in the center of the laser dot in case

230 of normal light incidence. Lastly, iii) the distance (PFC) between the center of the image and the

231 two identified brightest pixels is computed. Post-processing of the images is performed after the

232 flight and takes around 30 seconds per image.

\subsubsection{GNSS system}

234 The differential GNSS system consists of two NovAtel receivers: one used as master station

235 (flexpack6) and one as rover (OEM628 board). A NovAtel GPS-703-GGG pinwheel triple

236 frequency and GLONASS antenna is used as base station and an antcom (3G0XX16A4-XT-1-4-

237 Cert) dual frequency GPS and GLONASS flight antenna is used as rover station on the UAV.

238 Raw pseudoranges and carrier phase measurements are stored at $5 \mathrm{~Hz}$. The position solution is

239 post-processed using Leica Geomatic Office v 8.1 in kinematic mode. In post-processed mode, a

240 Kalman filter can be applied both in forward and backward direction for best position 
241 performance. The length of the GPS baseline affects the vertical and horizontal accuracy of the

242 drone position. Position error is expected to increase by 1-3 ppm (1-3 mm additional error per

$243 \mathrm{~km}$ of baseline).

\subsubsection{Payload controller}

246 Data acquired by the different sensors are saved on the SBC (BeagleBone Black) and a time

247 synchronization of the different sensors can be performed. Synchronization between the position

248 retrieved by the GNSS system and the range retrieved by the sensors is essential for accurate

249 water level observations, as described in Appendix B.

\subsection{Testing of the sensors}

252 To test the accuracy of the system, both static (ground-based) and dynamic (airborne) tests were

253 performed. First, several tests were conducted from bridges of different heights over free-flowing

254 rivers in order to test accuracy, precision and maximum ranging capability. Beam divergence

255 was tested by acquiring measurements inside a water well of small diameter. After the ground-

256 based tests, numerous flight tests were conducted over a lake. Because the water level in the lake

257 can be assumed to be uniform in space, these flights allowed determination of the accuracy of the

258 full system, which consists of the GNSS receiver and the ranging sensors. Appendix B reports

259 the experimental settings of both static and airborne tests. 
262 Accuracy of the ranging sensors was estimated using as reference a water level dip meter, which

263 has an accuracy better than $0.3 \%$ of the range. When tested in static mode, sensors acquired

264 measurements for 30 seconds. Subsequently the average range $(\bar{x})$ was computed as the weighted

265 arithmetic mean as shown in equation (7) after outlier removal $(\geq 5 \sigma)$.

$$
\bar{x}=\frac{\sum_{i=1}^{N} f_{i} x_{i}}{\sum_{i=1}^{N} f_{i}}
$$

267 In equation (7) $\mathrm{x}_{\mathrm{i}}$ is an observation and $\mathrm{f}_{\mathrm{i}}$ the frequency of that value. $\mathrm{N}$ is the total number of 268 measurements which depends on the reading range of the individual sensor.

269 Precision is estimated as standard deviation $(\sigma)$ of the measuring stack, and is computed using 270 equation (8):

$$
\sigma=\sqrt{\frac{\sum_{i=1}^{N} f_{i} \cdot\left(x_{i}-\bar{x}\right)^{2}}{\sum_{i=1}^{N} f_{i}-1}}
$$

272 Maximum ranging capability is the maximum range from which the sensor can retrieve a

273 measurement with a reasonable accuracy (i.e. 5\% of the range).

274 Beam divergence is defined as the measure (in angular units) of the increment in beam

275 diameter with distance from the optical aperture or antenna from which the sonic or

276 electromagnetic beam emerges. A larger beam divergence leads to a larger ground footprint of

277 the signal, which results in contamination of the signal if the surface is inhomogeneous. For the

278 CLDS this parameter is negligible, since its ground footprint directly depends on the arm length

279 A and the laser beam divergence is very low. Moreover, the CLDS provides images of each

280 individual acquisition and the user can perform a-posteriori supervision to control if the 
measured target is indeed the water surface. For the radar and the sonar, beam divergence is a

282 critical parameter to ensure that water is measured without interference from the surroundings.

283 This parameter has to be considered in order to monitor water bodies (e.g. large sinkholes, rivers

284 surrounded by dense vegetation), which only expose a narrow stretch of water to aerial view.

285 Indeed, because of loss of GNSS signal, flights under vegetation canopy or inside small cavities

286 (e.g. karst sinkholes) cannot be performed without losing position accuracy. Beam divergence

287 was estimated by acquiring measurements over water wells of small diameter, while water was

288 gradually being pumped out, as described in Appendix B.

\subsubsection{Airborne evaluation}

290 Numerous flights were conducted above a $0.02 \mathrm{~km}^{2}$ lake located near Holte, Denmark

$291\left(55.821720^{\circ} \mathrm{N}, 12.509067^{\circ} \mathrm{E}\right)$. Water level in the lake is practically uniform. Whilst the sonar 292 and the CLDS identify only one target in the field of view, the radar can identify multiple targets

293 and reports the target angle for each of those. This requires an accurate identification of the

294 target, which is representative of the water surface. Indeed, sometimes multiple targets are

295 retrieved at nadir angle, for instance when vegetation is overhanging the water body. In that case,

296 postprocessing requires switching between different targets to obtain a result that is continuous

297 in time. Moreover, a low-pass digital filter was applied on the $15 \mathrm{~Hz}$ raw radar data. A weighted

298 moving average (WMA) with a temporal window of $0.33 \mathrm{~s}$ (five observations) was applied to

299 smoothen the signal as shown in equation (9).

$$
W M A_{t}=w_{1} A_{t-2}+w_{2} A_{t-1}+w_{3} A_{t}+w_{4} A_{t+1}+w_{5} A_{t+2}
$$


301 Weights (w1, w2...w5) are normally set to a high value for the measurement taken at the actual

302 time (At) and to lower values for the previous and subsequent measurements.

303 .The overall accuracy of the system consisting of the GNSS receiver and the ranging sensor

$304\left(\sigma_{t o t}\right)$ is assumed to be that of two independent normally distributed variables: the ranging sensor

305 accuracy and the GNSS accuracy (10).

$$
\sigma_{t o t}=\sqrt{\sigma_{S}^{2}+\sigma_{R T K}^{2}}
$$

307 where $\sigma_{S}$ is the accuracy of the ranging sensor and $\sigma_{R T K}$ is the accuracy of the GNSS receiver.

\section{3. Results}

309 The first section of the results describes the technical performance of the ranging sensors when

310 tested from a static position on the ground. Results are based on numerous tests conducted from

311 bridges of different heights to compare the technical performance of the different sensors. The

312 second section describes the results of the flight tests that are intended to evaluate the accuracy

313 of the integrated system, i.e. GNSS receiver and sensors operating on board the UAV.

\subsection{Ground-based performance results}

315 Sensors demonstrated different performance in terms of accuracy and standard deviation of the

316 measuring stack when tested from bridges of different heights. Appendix B lists the experimental

317 settings for the static tests. Fig. 8 shows that the sonar usually tends to overestimate the range to

318 water surface, which is probably caused by a slight penetration of the ultrasonic wave (42 $\mathrm{kHz})$

319 below the water surface. Conversely, the radar usually tends to underestimate the range. The 
320 authors guess that this is due to the post-processing of the raw data by the proprietary radar

321 firmware.

324 Fig. 8

327 Table 1 summarizes the sensors' technical performance in terms of accuracy, standard deviation 328 of the measurement stack, maximum ranging distance and beam divergence.

332 Table 1 confirms that the sonar is the best sensor in terms of accuracy and standard deviation of

333 the measurement stack. The CLDS has the lowest beam divergence. However, the radar is the 334 sensor that combines the longest ranging capability, with accuracy and standard deviation that 335 are only slightly worse than for the sonar. In Fig.9, two regression lines confirm the systematic 336 error of radar and sonar. Plotted as function of the range, the regression line of the radar absolute 337 error has a slope of -0.0090 , while the slope of the sonar is 0.0083 . After removal of this

338 systematic error, the radar shows an accuracy of $0.5 \%$ of the range, whilst the accuracy of the 339 sonar is around $0.3 \%$. 


\section{$342 \quad$ Fig.9}

345 Finally, the accuracy of the retrieved vertical position has to be assessed. The accuracy of the

346 GNSS height depends mainly on: i) the integer ambiguity solution that has to be fixed to obtain

347 reliable observations, ii) the satellite geometry that affects the dilution of precision (DOP), iii)

348 multipath interference, especially because of signal reflection from the water surface.

\subsection{Airborne performance results}

351 In this section, we report the observations of two flights and we show a table summarizing the

352 entire dataset of flights over the lake. The range measured by each of the sensors and the altitude

353 retrieved by the GNSS are shown in Fig.10. The figure contains the entire dataset of observations

354 retrieved by the radar and sonar. Only not-a-number $(\mathrm{NaN})$ values are removed. The sonar

355 outputs $\mathrm{NaN}$ when the range exceeds the maximum range capability (10 m). For the CLDS, we

356 only reported the measurements retrieved from images in which the laser dots are clearly

357 identifiable on the water surface.

$359 \quad$ Fig. 10

361 Fig.10 shows an extremely high correlation (Pearson coefficient of 0.9991), between the GNSS

362 and the radar measurements, which indicates the consistency of our ranging technology. The

363 laser dots are generally distinguishable on the water surface only when the range to water surface

364 is less than $12-13 \mathrm{~m}$. Similarly, the sonar provided accurate measurements only when the UAV 
365 was hovering at low altitudes (less than $10 \mathrm{~m}$ from the water surface). Indeed, the radar and

366 sonar curves only overlap during these flight maneuvers.

367 In Fig. 11 we display the water level measured by the different sensors. Outliers $(>2 \sigma)$ were 368 removed.

$370 \quad$ Fig. 11

372 Mode value, mean and standard deviation of water level retrieved by each of the sensors are 373 reported in Table 2 under the column with flight date "04/04". The dispersion in water level 374 measurements retrieved by the system consisting of the radar and the GNSS receiver may be due 375 to multipath errors on the GNSS receiver. The cut-off angle for the elevation of the satellites, 376 which defines the angle below which GPS satellites are excluded, turned out to be a sensitive 377 parameter. The selected values for each flight are reported in Appendix B.

378 The water level values retrieved by the sonar had low accuracy, especially during high-speed 379 maneuvers. Since the range to water surface was greater than the maximum range capability of 380 the sonar for a significant portion of flight duration, the sonar retrieved many NaN values and 381 noisy observations. However, the mode value retrieved by the sonar is $24.14 \mathrm{~m}$, which is close to 382 the mean value retrieved by the radar.

383 The CLDS exhibits only few observations due to limited range capability and low frame rate.

384 Moreover, natural light conditions complicate the recognition of the laser dots on the water 385 surface.

386 In order to estimate the absolute accuracy of the sensors, results were compared to in-situ 387 measurements of water level. For the in-situ measurement, an additional accurate RTK (Real 
388 Time Kinematic) GNSS rover station was used, which was connected to a Danish GPS network.

389 The position was averaged over a period of one minute which resulted in $24.10 \mathrm{~m}$ above the

390 DVR90 geoid model (with an estimated accuracy of the GNSS rover station of around 5-6 cm).

391 For this flight, the accuracy of the radar is thus better than $5 \mathrm{~cm}$, the mode value of the sonar is

392 around $4 \mathrm{~cm}$ from the ground truth, while the mean value retrieved by the CLDS is within two

393 decimeters.

394 The second flight reported in Fig. 12 evaluated performance for higher drone altitude (up to 60

$395 \mathrm{~m})$ above the water surface.

397 Fig. 12

398 As shown in Fig. 12, the radar and the GNSS show very high correlation for the entire flight.

399 The flight confirmed the limited ranging capability of the sonar (specified as $10 \mathrm{~m}$, but already

400 very noisy beyond $9 \mathrm{~m}$ ). The CLDS retrieved ranges up to $13 \mathrm{~m}$, however standard deviations

401 increased significantly with range. In Fig. 13 we compare the water level retrieved by the three 402 different sensors for this flight.

404 Fig. 13

406 Statistics of the flight are shown in Table 2 under the column " $27 / 05$ ". In-situ water level was

40724.01 m. Fig. 13 shows that the sonar measurements were unsuccessful. The CLDS, despite very

408 high standard deviations, shows a mean value that is very close to the ground truth. The radar

409 shows higher dispersion for long ranges. Moreover, systematic error is still observable, in fact 
410 when the drone is at higher altitude, the retrieved water level increases by a few cm. System

411 performance was confirmed in a number of other flights, as shown in Table 2 . Experimental

412 settings, such as flight speed, illumination conditions, sensor settings for each flight, are

413 explained in appendix B.

414

415

416 Table 2

417

418 Table 2 clearly indicates that the radar is the most reliable sensor, with the lowest standard

419 deviation and good agreement with in-situ measurements. However, during some of the flights,

420 the measured water level exhibits significant standard deviation also for the radar. This

421 dispersion of the water level observations is caused not only by ranging errors but also by the

422 GNSS. Indeed, during some flights, the geometrical configuration of GNSS satellites may have

423 been suboptimal for accurate positioning. In addition to this, multipath of the GNSS signal may

424 occur and degrade the accuracy of water level observations to ca. $7 \mathrm{~cm}$.

425 The sonar provides very noisy measurements and exhibits a skewed distribution with a fat tail

426 around $10 \mathrm{~m}$, which is the maximum range of the sensor. While the mean value of water level

427 does not provide an accurate estimate, the mode values measured by the sonar are very similar to

428 the corresponding values measured by the radar.

429 For the CLDS, the mode value is not relevant because the number of observations is low. The

430 CLDS standard deviation is quite large and in order to obtain accurate results the drone has to

431 hover for several seconds. 


\section{4. Discussion}

433 The ranging technology showed great potential in terms of accuracy, maximum range and beam 434 divergence. In particular, the radar demonstrated the best performance in terms of accuracy and 435 maximum range. The ranging sensor has to be integrated with carrier phase differential GNSS to

436 retrieve water level. The accuracy of the integrated system consisting of GNSS receiver and 437 radar is estimated to be better than 5-7 $\mathrm{cm}$. This accuracy can be compared with the accuracy

438 achievable with: i) airborne LIDARs, ii) spaceborne laser altimetry, iii) spaceborne radar 439 altimetry and iv) ground-based stations as shown in Table 3.

Table 3

446 Few studies report the accuracy of LIDAR system in measuring water surface, but it has been 447 estimated to vary from few $\mathrm{cm}$ up to two tens of $\mathrm{cm}$ (Hopkinson et al., 2011). For airborne 448 LIDAR systems, the inaccuracy of the onboard positioning systems has to be included. Similarly 449 spaceborne laser altimetry from ICESat, which is the satellite altimeter with the smallest 450 footprint (50-90 m) and the highest along-track resolution $(40 \mathrm{~Hz}, 170 \mathrm{~m})$, provides water 451 surface elevation measurements for rivers with an accuracy at decimeter level. However the 452 accuracy degrades in case of cloud cover (Phan et al., 2012). Additionally, simultaneous return 453 from land and water are inevitable for small rivers and the identification of water surfaces 454 remains problematic. The accuracy of radar altimetry sensors such as the systems on board 
456 al., 2015) is in the order of some tens of $\mathrm{dm}$. Moreover, satellite radar altimetry generally has a

457 spatial resolution lower than satellite laser altimetry and requires that rivers are hundreds of

458 meters wide to avoid signal contamination by interfering land and vegetation (Maillard et al.,

459 2015). With UAV-borne monitoring, water surface and interfering surroundings can be clearly

460 separated due to the smaller ground footprint, and the possibility to retrieve individual radar

461 target angles. However, for very narrow fields of view, the CLDS is the only sensor that can

462 provide reliable water level measurements. Image analysis as part of the post-processing

463 workflow ensures that measurement are accepted only if the monitored target is the water

464 surface. This is the case for rivers surrounded by dense riparian vegetation or for small targets

465 such as karst sinkholes, e.g. on the Yucatán Peninsula (Gondwe et al., 2010). Our CLDS solution

466 overcomes the limitations of traditional red wavelength time-of-flight (TOF) laser distance

467 meters, which are not suitable for ranging to water surfaces, because the reflectivity of water is

468 very low for red visible wavelengths.

469 Only ground-based hydrometric stations ensure an accuracy higher than the one achieved with

470 UAV-based monitoring, but coverage and reliability of in-situ monitoring networks have been

471 degrading in many regions of the world. Moreover, despite providing high accuracy and

472 temporal resolution, in-situ stations acquire only local measurements and tend to fail during

473 extreme events. Therefore, UAV-based water level monitoring is beneficial for the monitoring of

474 a wide range of hydrological systems, including small-scale rivers, ephemeral lakes, sinkholes,

475 meltwater lakes, etc... UAV-based water level observations can resolve the spatial

476 multidimensional variability of rivers. Indeed, UAVs can monitor water level along and across

477 the river course, in order to obtain water slope and assess interaction between rivers and adjacent 
478 floodplains. Improved sharpness and reliability of estimates of surface water-groundwater

479 interaction using UAV-based monitoring of river water levels have already been reported

480 (Bandini et al., 2016). Furthermore, UAVs can sense water level in unconventional remote

481 sensing targets such as sinkholes or cenotes. This could potentially improve mapping of phreatic

482 surfaces, for instance for the Yucatan peninsula (Bauer-Gottwein et al., 2011). Additionally,

483 UAVs can potentially be used during extreme events when in-situ monitoring stations often fail

484 and satellite observations do not ensure the required spatial and temporal resolution. Thus, UAVs

485 have the potential to improve flood risk assessment. However, the $\pm 7 \mathrm{~cm}$ accuracy of our

486 technology may still be insufficient for rivers flowing through low-lying terrain. Nonetheless, the

487 accuracy is better than other spaceborne and airborne technologies and UAVs have a great

488 potential in improving flood mapping because they allow optimal timing of the observations and

489 high spatial resolution. UAV-based observations of water level in the flooded areas allow

490 determination of stage-damage curves (Cammerer et al., 2013) which are essential for the design

491 of insurance policies.

\section{5. Conclusions}

493 UAV-based remote sensing of river and lake water level (orthometric height) has the potential to 494 fill the gap between in-situ measurements and spaceborne remote sensing. It ensures: i) high

495 accuracy, ii) optimal spatial resolution, iii) flexible timing of the sampling, and iv) precise 496 tracking of lakes and rivers. Different water surface ranging sensors were tested: a radar, a sonar, 497 and a CLDS.

498 Static (on ground) and dynamic (airborne) tests demonstrated the following results: 
- The radar showed the best accuracy and longest maximum range. Despite having a resolution of only $10 \mathrm{~cm}$, averaging the $15 \mathrm{~Hz}$ primary data, an accuracy of $0.5 \%$ of the range can be achieved after correction of a negative bias of $0.9 \%$ of the range.

- The sonar provided unreliable results for high ranges or high speeds. Our results show that the sonar generally overestimates the range to water surface. However, when the UAV flies at a stable and low height, the accuracy is down to a few centimeters.

- The CLDS is less accurate than the radar. However, it has the lowest beam divergence and is useful when only a narrow field of view to the water surface is available for sensing.

Water level can be measured on board UAVs by subtracting the range to water surface from the vertical position retrieved by the GNSS receiver. Dynamic (airborne) tests have been performed on the positioning technology and the GNSS receiver had a vertical accuracy

514 The integrated system GNSS receiver and radar is able to measure water level with an overall 515 accuracy better than 5-7 $\mathrm{cm}$ when the UAV flies at a speed of few $\mathrm{km} / \mathrm{h}$.

516 Future research should include different types of sonar sensors, trading off signal penetration 517 below the water surface (more penetration at lower frequencies) and interference of the propeller 518 noise (more interference at higher frequencies). Moreover, research efforts are ongoing to 519 develop new radars with higher measurement resolution, exploiting other region of the 520 microwave spectrum commonly used in radar altimetry such as $\mathrm{Ku}$ and $\mathrm{Ka}$ bands. 
524 The master thesis from Reyna Gutierrez, J. A. (2013) '’Monitoring and modeling of regional

525 groundwater flow on the Yucatán Peninsula' can be obtained from the authors upon request.

\section{Acknowledgements}

527 The Innovation Fund Denmark is acknowledged for providing funding for this study via the 528 project Smart UAV [125-2013-5].

\section{References}

530

Alsdorf, D.E., Rodriguez, E., Lettenmaier, D.P., 2007. Measuring surface water from space. Rev. Geophys. 45, 1-24. doi:10.1029/2006RG000197.1

Anderson, K., Gaston, K.J., 2013. Lightweight unmanned aerial vehicles will revolutionize spatial ecology. Front. Ecol. Environ. 11, 138-146. doi:10.1890/120150

Asadzadeh Jarihani, A., Callow, J.N., Johansen, K., Gouweleeuw, B., 2013. Evaluation of multiple satellite altimetry data for studying inland water bodies and river floods. J. Hydrol. 505, 78-90. doi:10.1016/j.jhydrol.2013.09.010

Bandini, F., Butts, M., Vammen Torsten, J., Bauer-Gottwein, P., 2016. Water level observations from Unmanned Aerial Vehicles (UAVs) for improving probabilistic estimations of interaction between rivers and groundwater. Geophys. Res. Abstr. 18, EGU2016-3144.

Bauer-Gottwein, P., Gondwe, B.R.N., Charvet, G., Marin, L.E., Rebolledo-Vieyra, M., MeredizAlonso, G., 2011. Review: The Yucatan Peninsula karst aquifer, Mexico. Hydrogeol. J. 19, 507-524. doi:10.1007/s10040-010-0699-5

Birkett, C.M., 1998. Contribution of the TOPEX NASA Radar Altimeter to the global monitoring of large rivers and wetlands. Water Resour. Res. 34, 1223. doi:10.1029/98WR00124

Birkett, C.M., Mertes, L. a K., Dunne, T., Costa, M.H., Jasinski, M.J., 2002. Surface water dynamics in the Amazon Basin: Application of satellite radar altimetry. J. Geophys. Res. Atmos. 107, LBA-26. doi:10.1029/2001JD000609

Calmant, S., Seyler, F., Cretaux, J.F., 2008. Monitoring continental surface waters by satellite altimetry. Surv. Geophys. 29, 247-269. doi:10.1007/s10712-008-9051-1

Cammerer, H., Thieken, A.H., Lammel, J., 2013. Adaptability and transferability of flood loss 
functions in residential areas. Nat. Hazards Earth Syst. Sci. 13, 3063-3081. doi:10.5194/nhess-13-3063-2013

Colomina, I., Molina, P., 2014. Unmanned aerial systems for photogrammetry and remote sensing: A review. ISPRS J. Photogramm. Remote Sens. 92, 79-97. doi:10.1016/j.isprsjprs.2014.02.013

Danko, T., 2004. Webcam Based DIY Laser Rangefinder [WWW Document]. URL https://sites.google.com/site/todddanko/home/webcam_laser_ranger (accessed 11.9.15).

Detert, M., Weitbrecht, V., 2015. A low-cost airborne velocimetry system: proof of concept. J. Hydraul. Res. 53, 532-539. doi:10.1080/00221686.2015.1054322

Featherstone, W.E., 2001. Absolute and relative testing of gravimetric geoid models using Global Positioning System and orthometric height data. Comput. Geosci. 27, 807-814. doi:10.1016/S0098-3004(00)00169-2

Frappart, F., Calmant, S., Cauhopé, M., Seyler, F., Cazenave, A., 2006. Preliminary results of ENVISAT RA-2-derived water levels validation over the Amazon basin. Remote Sens. Environ. 100, 252-264. doi:10.1016/j.rse.2005.10.027

Gondwe, B.R.N., Lerer, S., Stisen, S., Marín, L., Rebolledo-Vieyra, M., Merediz-Alonso, G., Bauer-Gottwein, P., 2010. Hydrogeology of the south-eastern Yucatan Peninsula: New insights from water level measurements, geochemistry, geophysics and remote sensing. J. Hydrol. 389, 1-17. doi:10.1016/j.jhydrol.2010.04.044

Hale, G.M., Querry, M.R., 1973. Optical Constants of Water in the 200-nm to 200-microm Wavelength Region. Appl. Opt. 12, 555-563. doi:10.1364/AO.12.000555

Hopkinson, C., Crasto, N., Marsh, P., Forbes, D., Lesack, L., 2011. Investigating the spatial distribution of water levels in the Mackenzie Delta using airborne LiDAR. Hydrol. Process. 25, 2995-3011. doi:10.1002/hyp.8167

Kiel, B., Alsdorf, D., LeFavour, G., 2006. Capability of SRTM C- and X-band DEM Data to Measure Water Elevations in Ohio and the Amazon. Photogramm. Eng. Remote Sens. 72, 313-320. doi:10.14358/PERS.72.3.313

Kleinherenbrink, M., Ditmar, P.G., Lindenbergh, R.C., 2014. Retracking Cryosat data in the SARIn mode and robust lake level extraction. Remote Sens. Environ. 152, 38-50. doi:10.1016/j.rse.2014.05.014

Kleinherenbrink, M., Lindenbergh, R.C., Ditmar, P.G., 2015. Monitoring of lake level changes on the Tibetan Plateau and Tian Shan by retracking Cryosat SARIn waveforms. J. Hydrol. 521, 119-131. doi:http://dx.doi.org/10.1016/j.jhydrol.2014.11.063

Lawford, R., Strauch, A., Toll, D., Fekete, B., Cripe, D., 2013. Earth observations for global water security. Curr. Opin. Environ. Sustain. 5, 633-643. doi:10.1016/j.cosust.2013.11.009

Lee, H., Beighley, R.E., Alsdorf, D., Jung, H.C., Shum, C.K., Duan, J., Guo, J., Yamazaki, D., Andreadis, K., 2011. Characterization of terrestrial water dynamics in the Congo Basin using GRACE and satellite radar altimetry. Remote Sens. Environ. 115, 3530-3538. doi:10.1016/j.rse.2011.08.015 
LeFavour, G., Alsdorf, D., 2005. Water slope and discharge in the Amazon River estimated using the shuttle radar topography mission digital elevation model. Geophys. Res. Lett. 32, L17404. doi:10.1029/2005GL023836

Lejot, J., Delacourt, C., Piégay, H., Fournier, T., Trémélo, M.-L., Allemand, P., 2007. Very high spatial resolution imagery for channel bathymetry and topography from an unmanned mapping controlled platform. Earth Surf. Process. Landforms 32, 1705-1725. doi:10.1002/esp. 1595

Luo, C., Nightingale, J., Asemota, E., Grecos, C., 2015. A UAV-Cloud System for Disaster Sensing Applications, in: 2015 IEEE 81st Vehicular Technology Conference (VTC Spring). IEEE, pp. 1-5. doi:10.1109/VTCSpring.2015.7145656

Maillard, P., Bercher, N., Calmant, S., 2015. New processing approaches on the retrieval of water levels in Envisat and SARAL radar altimetry over rivers: A case study of the São Francisco River, Brazil. Remote Sens. Environ. 156, 226-241. doi:10.1016/j.rse.2014.09.027

Phan, V.H., Lindenbergh, R., Menenti, M., 2012. ICESat derived elevation changes of Tibetan lakes between 2003 and 2009. Int. J. Appl. Earth Obs. Geoinf. 17, 12-22. doi:10.1016/j.jag.2011.09.015

Reyna Gutierrez, J.A., 2013. Monitoring and modeling of regional groundwater flow on the Yucatán Peninsula. Unpubl. master's thesis, Tech. Univ. Denmark.

Rodriguez, E., Morris, C., Belz, J., 2006. An assessment of the SRTM topographic products. Photogramm. Eng. Remote Sensing 72, 249-260. doi:0099-1112/06/7203-0249/\$3.00/0

Schumann, G., Matgen, P., Cutler, M.E.J., Black, a., Hoffmann, L., Pfister, L., 2008. Comparison of remotely sensed water stages from LiDAR, topographic contours and SRTM. ISPRS J. Photogramm. Remote Sens. 63, 283-296. doi:10.1016/j.isprsjprs.2007.09.004

Schutz, B.E., Zwally, H.J., Shuman, C. a., Hancock, D., DiMarzio, J.P., 2005. Overview of the ICESat mission. Geophys. Res. Lett. 32, 1-4. doi:10.1029/2005GL024009

Song, C., Ye, Q., Sheng, Y., Gong, T., 2015. Combined ICESat and CryoSat-2 altimetry for accessing water level dynamics of Tibetan lakes over 2003-2014. Water (Switzerland) 7, 4685-4700. doi:10.3390/w7094685

Tauro, F., Petroselli, A., Arcangeletti, E., 2015. Assessment of drone-based surface flow observations. Hydrol. Process. 30, 1114-1130. doi:10.1002/hyp.10698

Tauro, F., Porfiri, M., Grimaldi, S., 2016. Surface flow measurements from drones. J. Hydrol. 540, 240-245. doi:10.1016/j.jhydrol.2016.06.012

Villadsen, H., Andersen, O.B., Stenseng, L., Nielsen, K., Knudsen, P., 2015. CryoSat-2 altimetry for river level monitoring - Evaluation in the Ganges-Brahmaputra River basin. Remote Sens. Environ. 168, 80-89. doi:10.1016/j.rse.2015.05.025

Watts, A.C., Ambrosia, V.G., Hinkley, E.A., 2012. Unmanned Aircraft Systems in Remote Sensing and Scientific Research: Classification and Considerations of Use. Remote Sens. 4(6), 1671-1692. doi:10.3390/rs4061671 
631 Wingham, D.J., Francis, C.R., Baker, S., Bouzinac, C., Brockley, D., Cullen, R., de Chateau-

632

633

634

635

636

637

638

639

640

641

642

643

\section{Appendix A. Calibration of the CLDS}

The CLDS needs to be calibrated in order to provide a ranging measurement. Calibration has

646 been performed acquiring multiple range measurements (from 0 to $12 \mathrm{~m}$ ) using a black vertical

647 wall as calibration target. Since the focal length of the camera is not exactly known, equation (4)

648 must be used and the calibration is used to retrieve the coefficients $r_{p p 1}$ and $r_{0}$ for converting from

649 pixel units to angular units. The relationships between $\theta^{\prime}$ and the distance from the laser dots to

650 the center of the image (PFC) are shown in Fig. A.1 for each of the laser pointers. Alternatively,

651 Fig. A.2 depicts the relationship between the range to the target and PFC. 
653 Fig. A.1 and Fig. A.2 show that the laser pointers' curves are not coincident as a consequence of

654 the slight asymmetry of the layout (imaging sensor of the camera not placed exactly in the 655 middle of the two laser pointers). As confirmed by Fig. A.1, the relationship between PFC and 656 the measured angle is approximately linear for each of the two laser pointers. Calibration has 657 shown an $\mathrm{r}$ (Pearson linear correlation coefficient of determination) of 0.99978 and an RMSE 658 (Root Mean Square Error) of $7.16 \mathrm{~cm}$ for the blue laser (left laser); an r of 0.99937 and an RMSE 659 of $8.29 \mathrm{~cm}$ for the green laser (right laser). Calibration error is displayed in Fig. A.3.

660 Fig. A.3

661

662 Fig. A.4

664 Fig. A.3 demonstrates that the advantage of using two laser pointers is improved error 665 assessment. Considering the average of the measurements of the two laser pointers, calibration 
666 RMSE is reduced to $5.61 \mathrm{~cm}$. When range to water surface has to be retrieved, the precise 667 computation of PFC is more problematic than during the simple calibration procedure. Indeed, 668 while laser dots can be normally identified as in Fig. A.4 (a), laser dots on the water surface 669 might have contours that are less defined as in Fig. A.4 (b). Sometimes even multiple laser dots 670 are visible, as shown in Fig. A.4 (c). This is caused by: i) atmospheric scattering processes, ii)

671 scattering processes due to water waviness iii) vibrations of the UAV. The laser light reflected

672 from the bottom is occasionally visible in the image, especially in case of shallow or very clear 673 water, as shown in Fig. A.4 (d). Experiments showed that the uncertainty in the PFC increases 674 with the range to water surface. This is displayed in Fig. A.5 with the curve PFC- $\sigma_{\text {PFC. Fig. A.5 }}$

675 clearly shows that the green laser exhibits larger uncertainty than the blue laser since green

676 wavelengths are scattered to a greater extent than blue wavelengths. The expected uncertainty in 677 the range can be estimated using the derivative of the range function as shown in equation (A.1).

$$
\sigma(\text { range })=\frac{\text { drange }}{\partial(P F C)} \sigma(P F C)
$$


Fig. A.5

Fig. A.6

678 Fig. A.6 shows that the uncertainty of the range estimate increases with the range to water 679 surface. This is a consequence of: i) the derivative of the curve in Fig. A.2 that increases in 680 absolute value for longer ranges (small inaccuracy in PFC determines high imprecision in the 681 range observation). The derivative is lower in absolute terms for the blue laser, because of the 682 prototype layout. ii) Increasing uncertainty of the PFC with increasing range (i.e. decreasing 683 PFC).

684

685

686 Appendix B. Experimental settings

687

688 
689 In Table B.1 we report the location, the date and time of the day, the environmental conditions

690 and the water flow speed for each of the static tests. The mean value and the standard deviation

691 of the measurements are shown in Figure 8.

692 Here Table B.1

693 Illumination conditions are reported in the table because they affect visibility of the laser dots on

694 the water surface. This factor has been critical only in case of sun glint conditions during which

695 laser dots are hardly identifiable. On the other hand, wind stress and current can affect water

696 surface roughness and change the intensity of the backscattered radar signal.

697 Estimates of beam divergence for the different sensors were obtained from tests above a

698 cylindrical water well of diameter (D) equal to $0.7 \mathrm{~m}$. The sensors were placed exactly in the

699 middle of the water well as shown in Fig. B.1. The initial range between the sensors and the

700 water surface was $0.5 \mathrm{~m}$. Subsequently, the well was pumped to gradually increase the range to

701 the water surface. Beam divergence $(\varphi)$ was then computed according to equation B.1.

$$
\varphi=2 * \tan ^{-1} \frac{D}{2 \cdot r_{c}}
$$

702 In equation B.1, $r_{c}$ is the critical range i.e. the range at which the sensor first produced erroneous

703 results because of interference with the well walls. Fig. B.1 provides an illustration of the

704 experimental setup.

706 Fig. B.1 
708 While the CLDS was able to retrieve the range to water surface for all water levels (beam width

709 is constant and equal to the arm length), the beam divergences of the radar and the sonar were

710 estimated using this method. For the radar, interferences started to occur at a range of $1.3 \mathrm{~m}$, and

711 for the sonar at $1 \mathrm{~m}$. Equation B.1, then gives beam divergence of the radar as ca. $30^{\circ}$ and beam

712 divergence for the sonar as ca. $40^{\circ}$.

713 Table B.2 shows the flight records for the tests conducted over the lake to estimate the airborne

714 accuracy of the system.

716 Table B.2

719 As Table B.2 shows, the GNSS satellite cut-off angle settings are different between the flights.

720 The cut-off angle showed an influence on the position accuracy, and thus on the water level

721 measurements, up to 1-2 cm. Larger cut-off angles reduce the number of satellites in the field of

722 view of the GNSS antenna, while smaller cut-off angles might increase multi-path effects (e.g.

723 GNSS signal reflected by the water surface).

724 Average and vertical speed was varied between the different flights to test the synchronization

725 between the GNSS system and the different sensors. Indeed, since water level is constant in the

726 lake, when the drone rapidly changes its altitude, equivalent variations should be recorded by the

727 ranging sensors and the GNSS system. Synchronization between the radar, sonar and the GNSS

728 was obtained at the $30 \mathrm{~ms}$ level, while synchronization with the CLDS was obtained at the $0.2 \mathrm{~s}$ 
729 level. The synchronization between the GNSS system and the CLDS is slightly worse because,

730 even though the SBC registers the time at which the camera is triggered, the actual time that the

731 CMOS camera needs to take a picture is unknown.

732

733

734

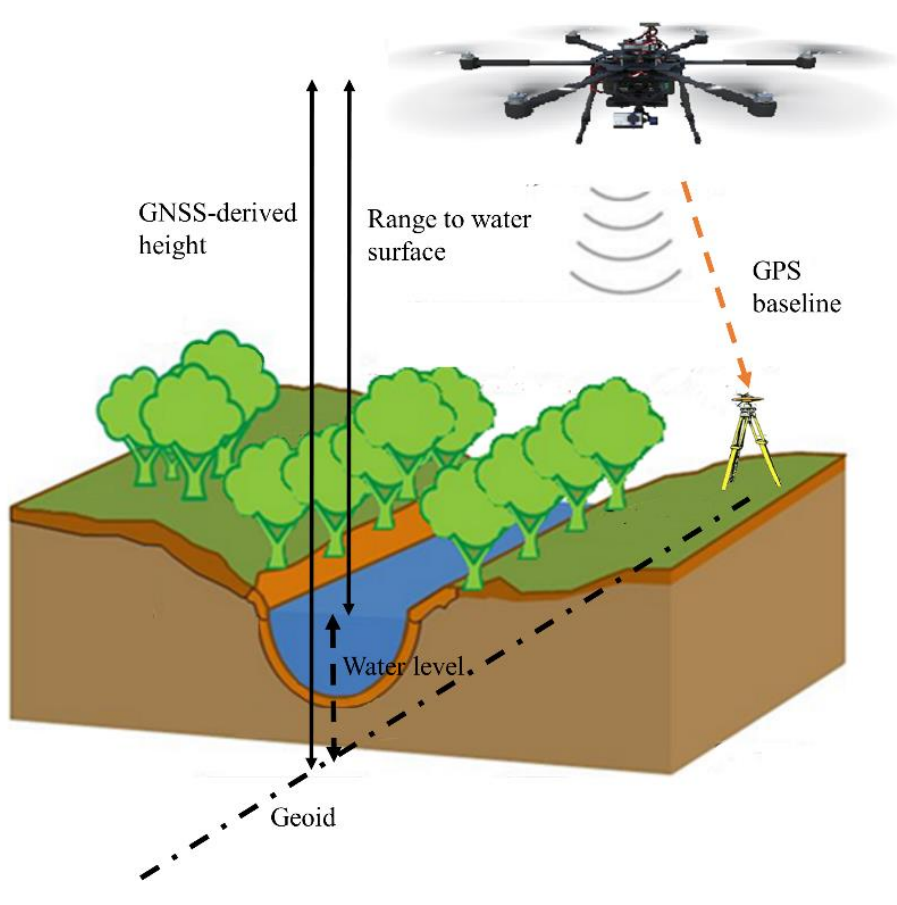

Fig. 1. Illustration of measurement principle for retrieving water level. The system includes: i) the UAV, ii) the sensors to measure the range from the UAV to the water surface, iii) a GNSS receiver on board the UAV providing accurate vertical and horizontal position. Centimeter-level position accuracy is obtained through the installation of an in-situ GNSS master station providing corrections for a kinematic post-processed solution. 
Fig. 2. Picture of the drone payload. It includes the three tested sensors (CLDS, radar and sonar), the GNSS system (antenna and receiver), the IMU, the Single Board Computer (SBC) and the power convertion units (DC/DC converters).

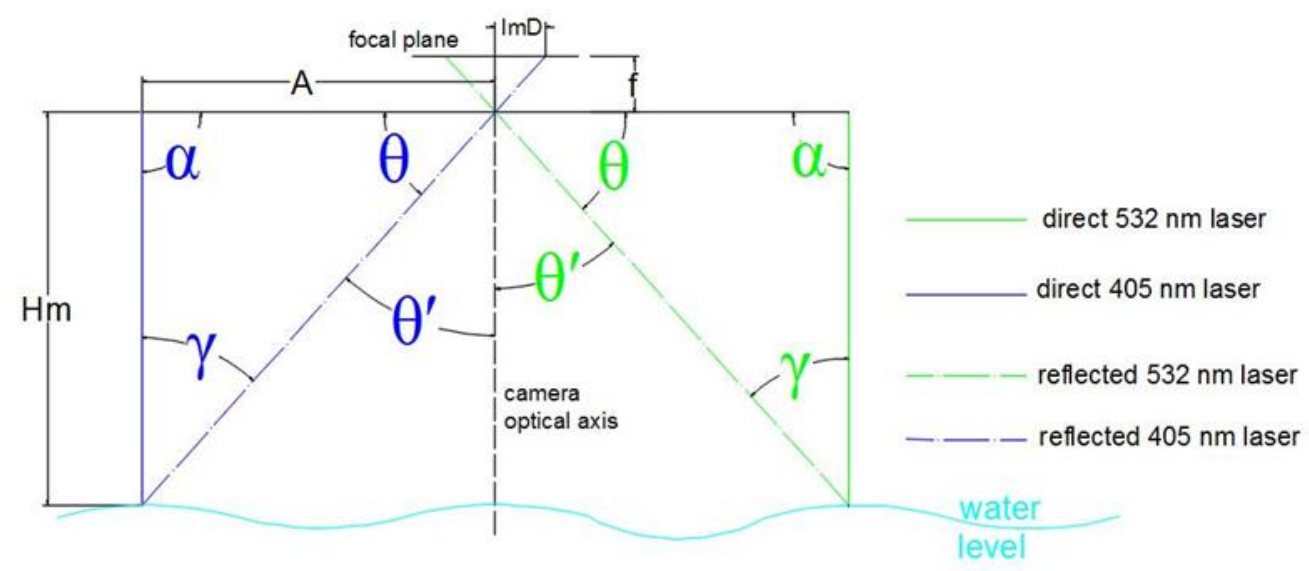

Fig. 3. Geometric configuration of the CLDS solution. A is the distance between the center of the camera and each of the laser pointers. $\alpha$ is the angle between each of the lasers and the focal plane of the camera. Hm is the distance between the camera and the water surface. $\operatorname{ImD}$ is the distance between the center of the image focal plane and each of the recorded laser light dots. $f$ is the focal length of the camera. $\theta^{\prime}$ is the reflection angle. $\theta$ is its angle between the axis of the CLDS and the reflected ray. $\gamma$ is the angle between incident and reflected ray. If $\alpha$ is $90^{\circ}$ (as in the 


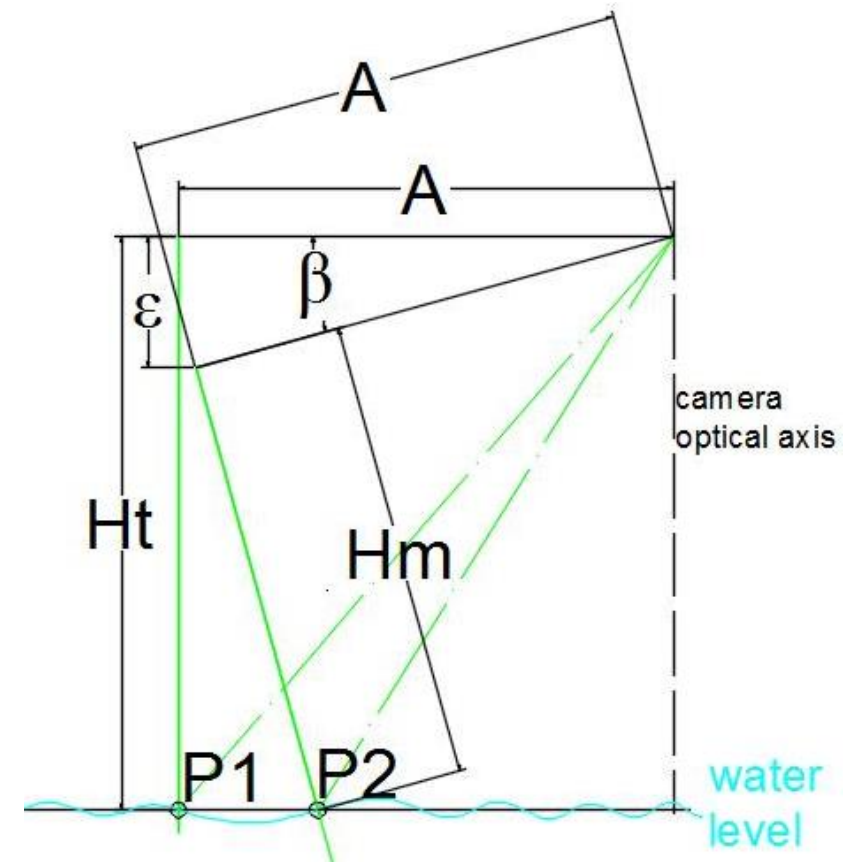

761

Fig. 4. Tilting angle: $\beta$ is the angle between the horizontal line and the plane where the lasers and the camera are located.

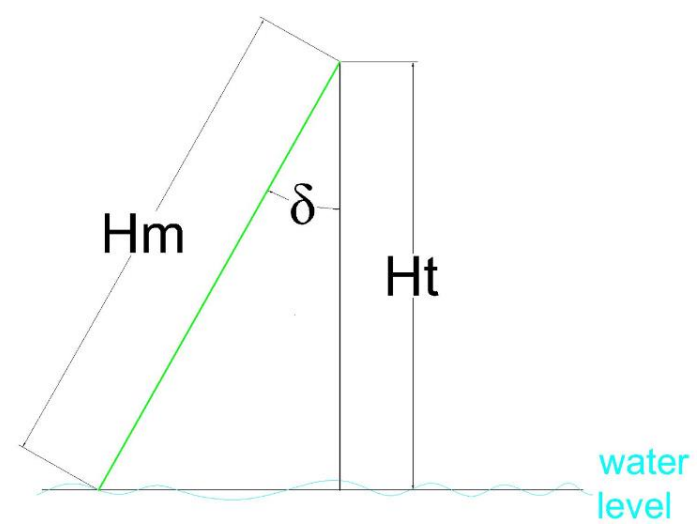




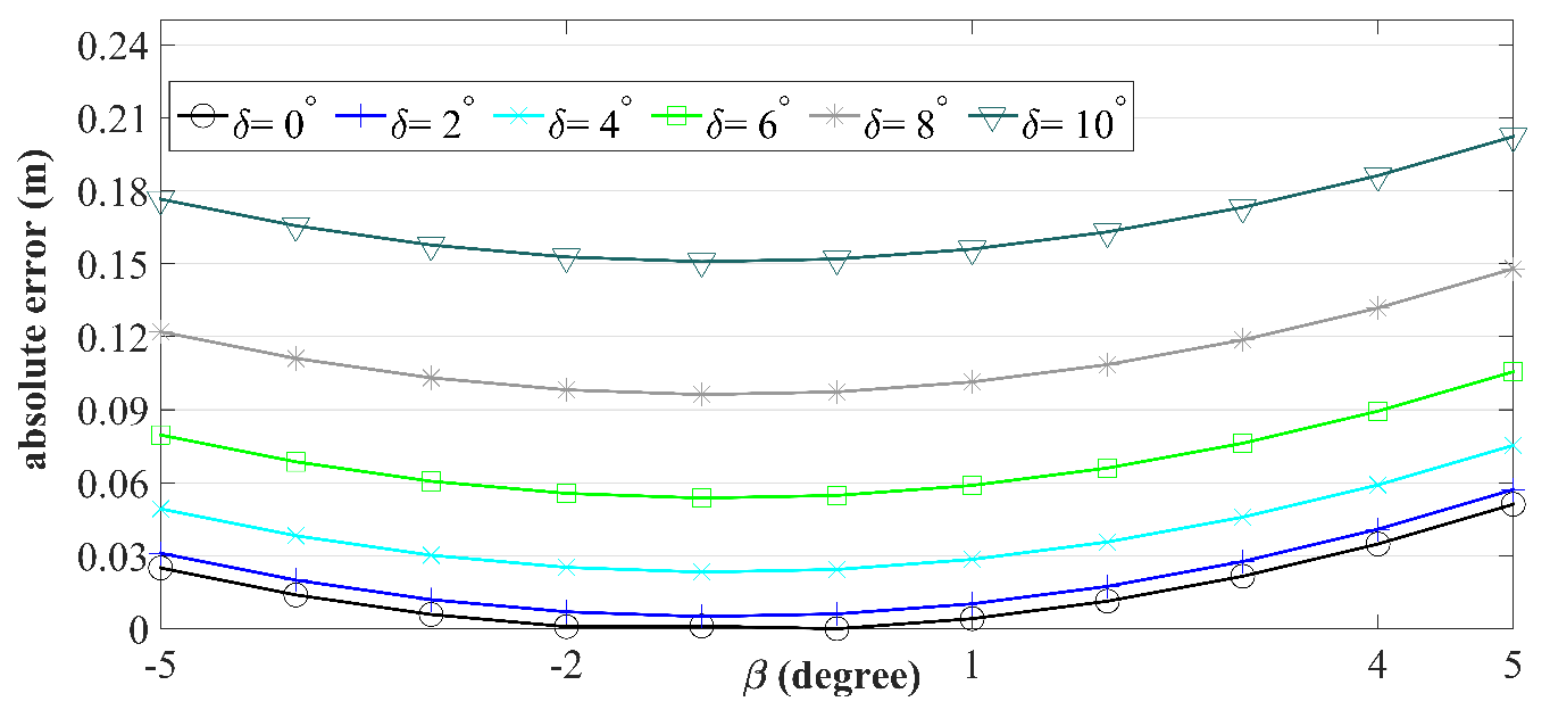

772

773

774

775

Fig. 6. Error as a function of displacement angles. Absolute error is shown for different tilting $(\beta)$ and rotation $(\delta)$ angles at $10 \mathrm{~m}$ range.

776

777

778

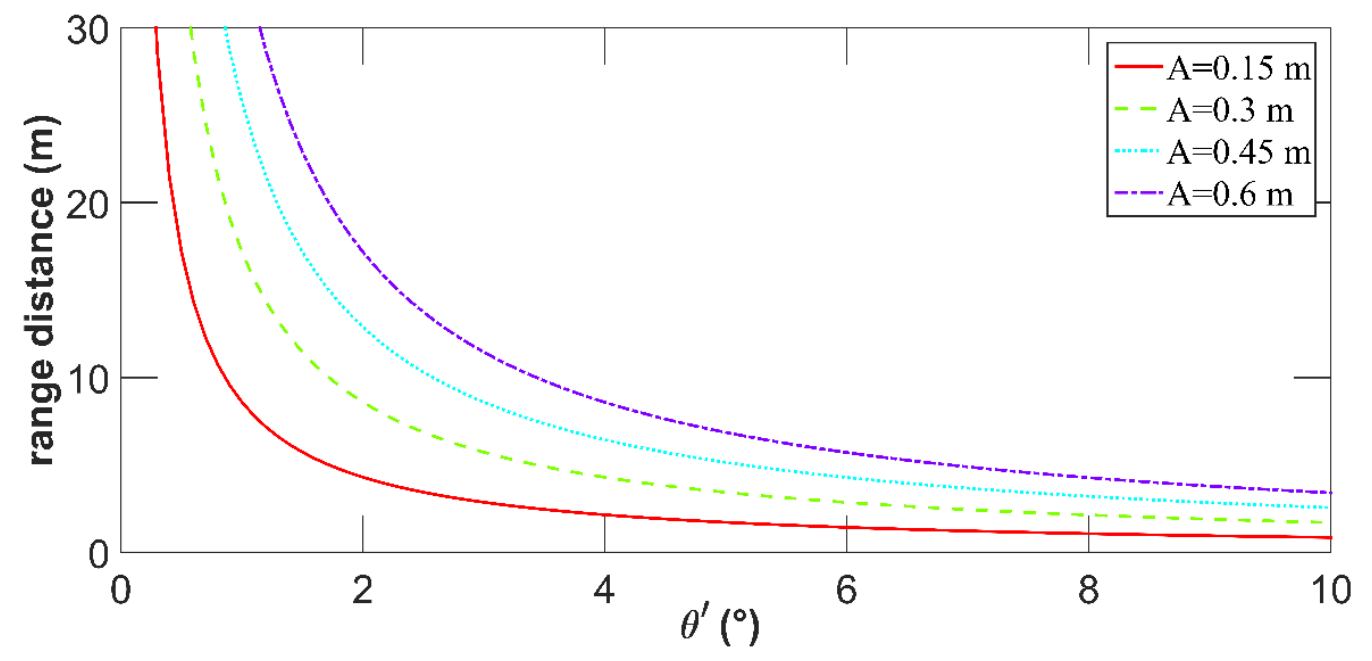

Fig. 7. Range distance as a function of the $\theta^{\prime}$ angle. The range function depends on the different length values of $A$, which is the distance between the laser source and the camera. 


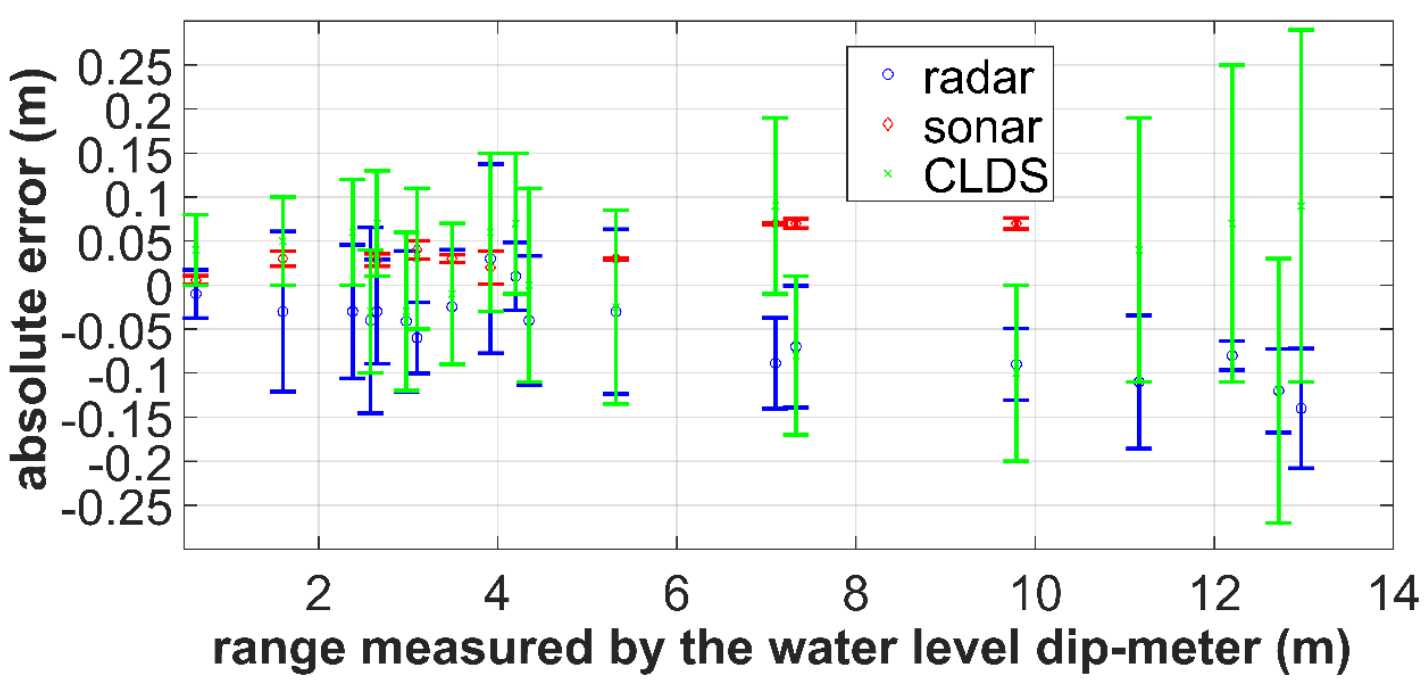

Fig. 8. Absolute error as a function of the range measured by each of the ranging sensors. Absolute error is computed using the water level dip meter as reference. The marker is the average error (bias) of all measurements taken for a specific range, while the bar shows the standard deviation.

790

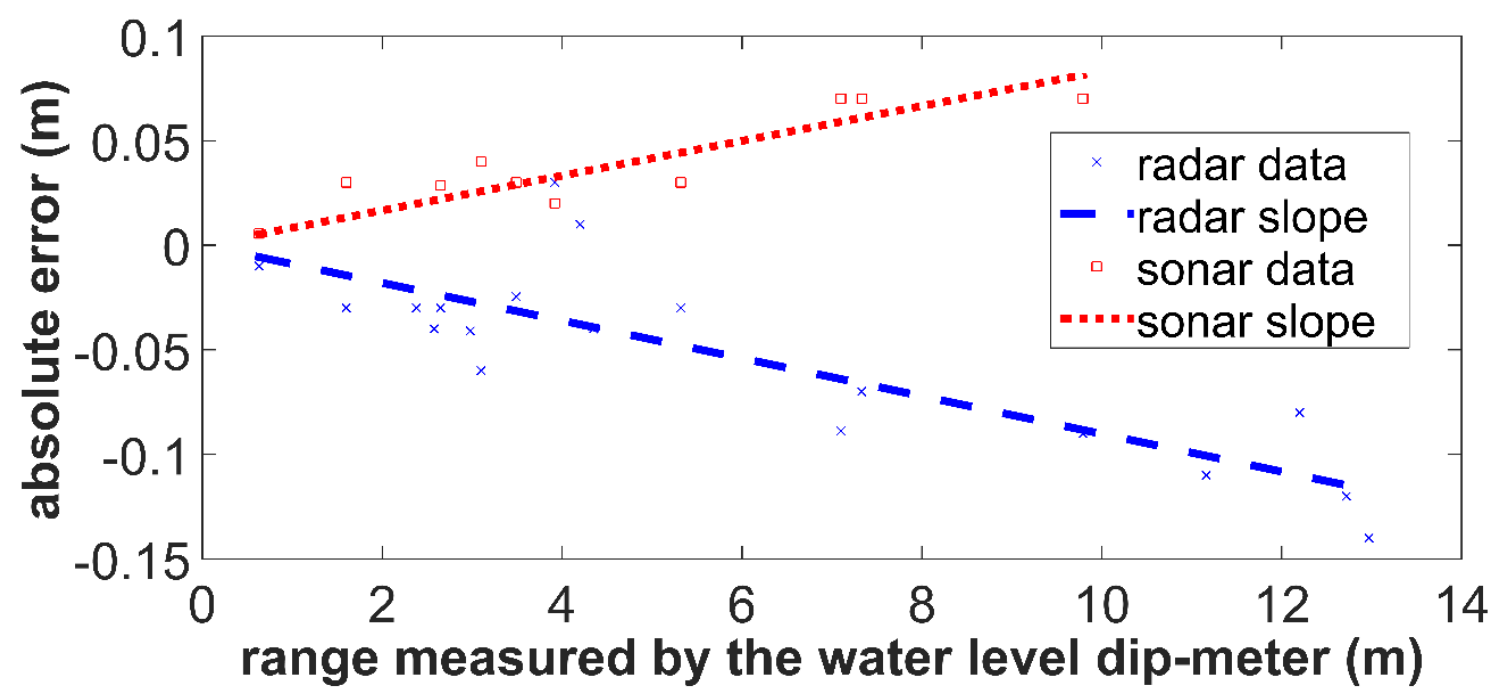

792

Fig. 9. Sonar and radar errors as a function of the range. Dots represent the measurements acquired by the radar and the sonar. The regression line shows that the absolute error is a function of the range. 


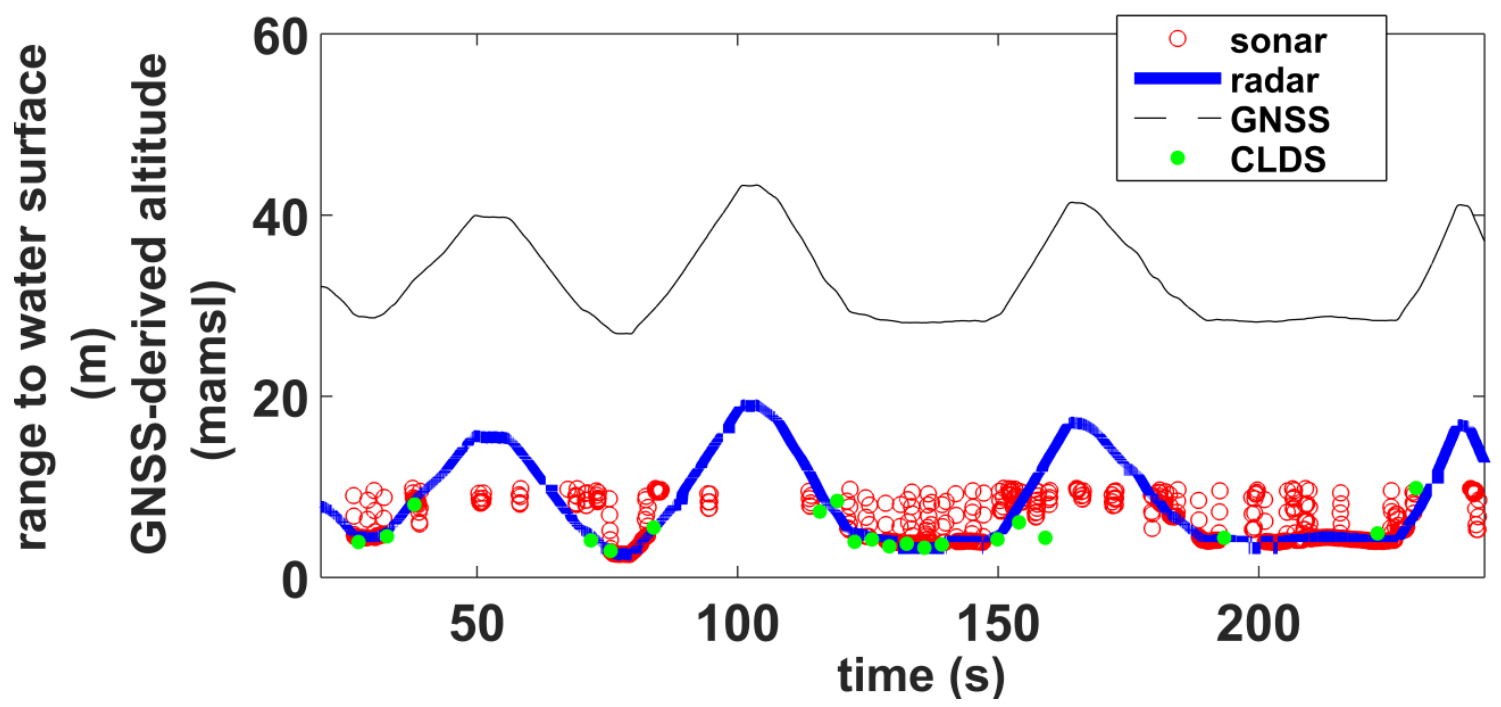

800

Fig. 10. Observations retrieved during the flight on April 4, 2016. The plot shows the range measured by the radar (blue), sonar (red), CLDS (green) in meter (m) to the water surface, and the drone altitude retrieved by the GNSS

[GNSS altitude-radar range]
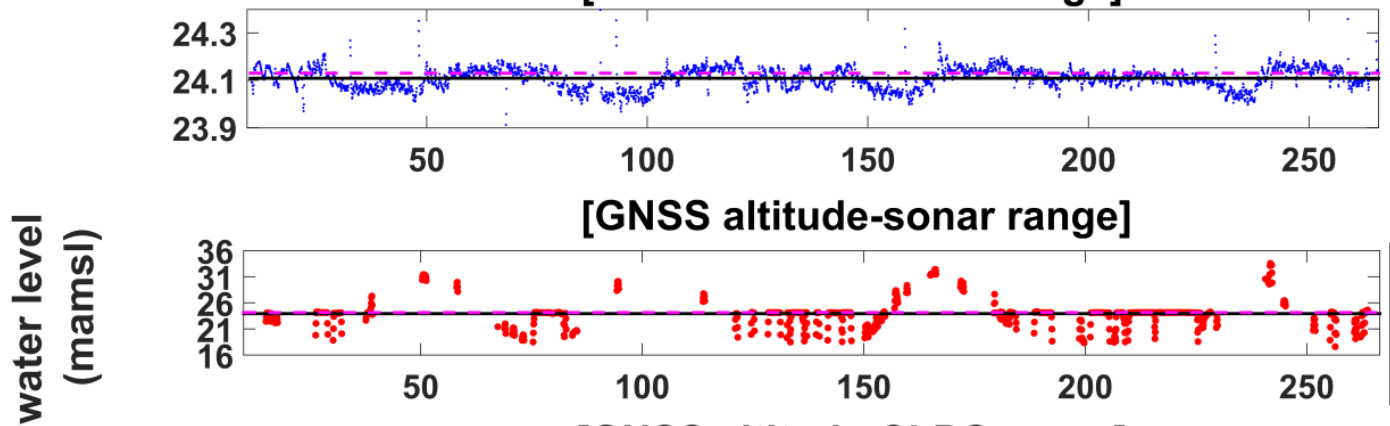

[GNSS altitude-sonar range]

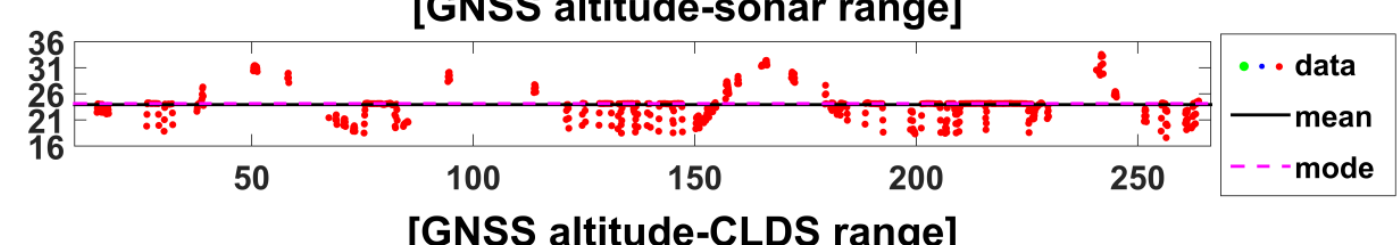

[GNSS altitude-CLDS range]

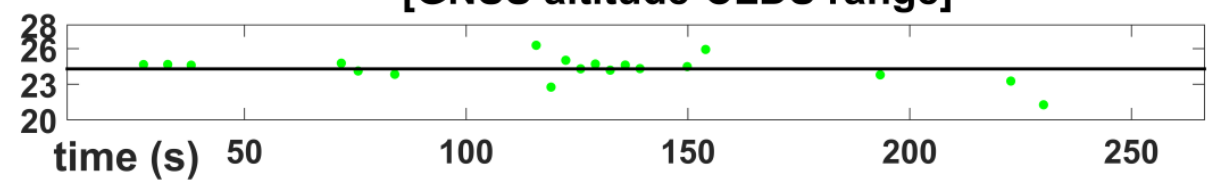

Fig. 11. Water level (mamsl) observations retrieved during the flight on April 4, 2016. Each of plots shows the water 


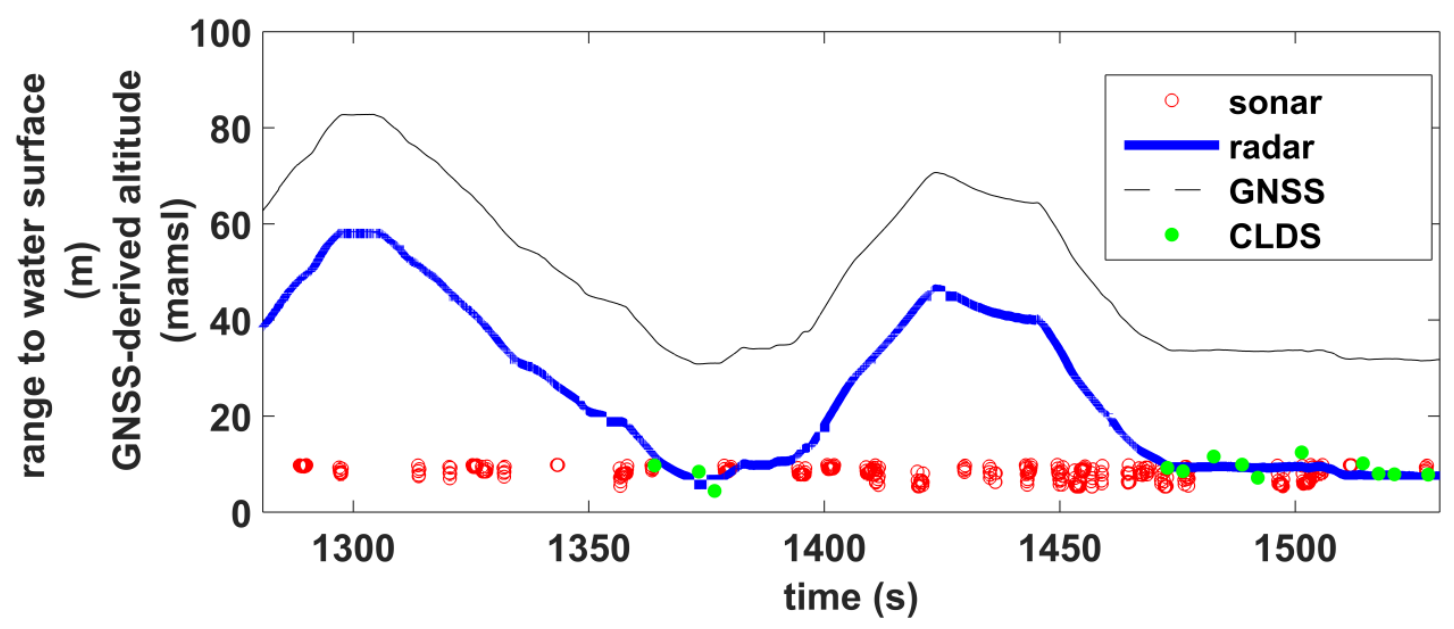

812

813 Fig. 12. Observations retrieved during the flight on May 27, 2016. The plot shows the range measured by the radar 814 (blue), sonar (red), CLDS (green) in meter (m) to the water surface, and the drone altitude retrieved by the GNSS 815 (black) in meter above mean sea level (mamsl).

816

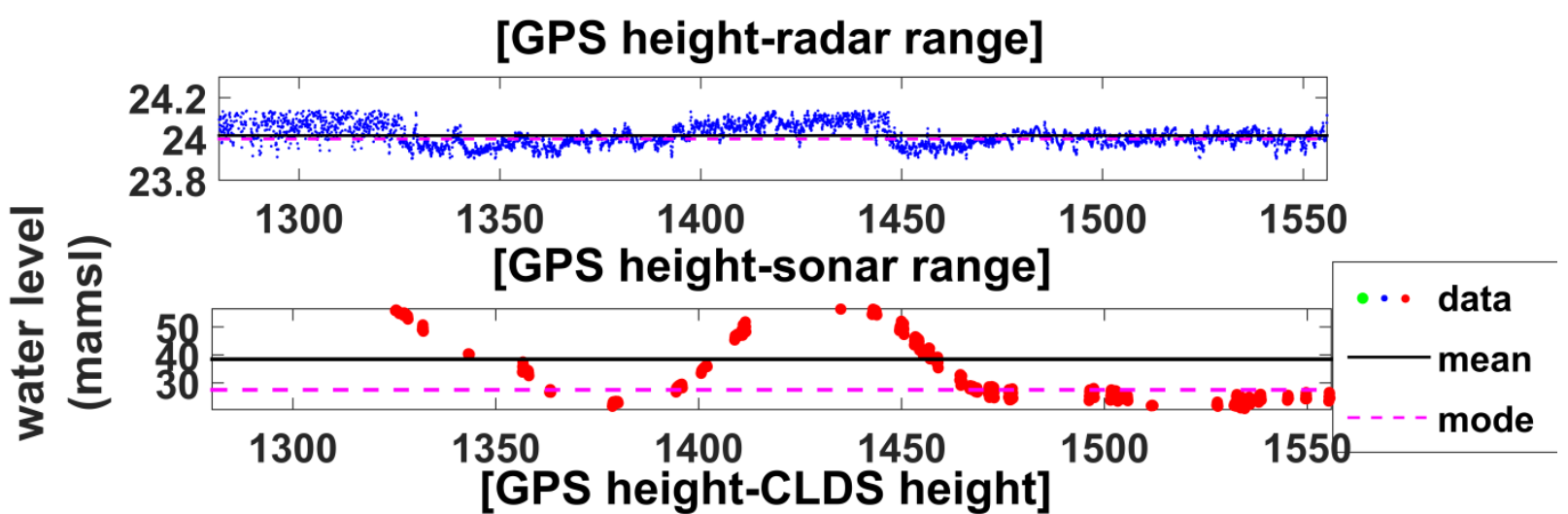

[GPS height-CLDS height] water level observations measured by subtracting the range retrieved by each of the sensors (radar, sonar, CLDS) from the GNSS-derived altitude. In each plot, the black line is the mean of the water level observations and the magenta line is the mode of those observations. 
826

827

828

829

830

831

832

833

834

835

836

837

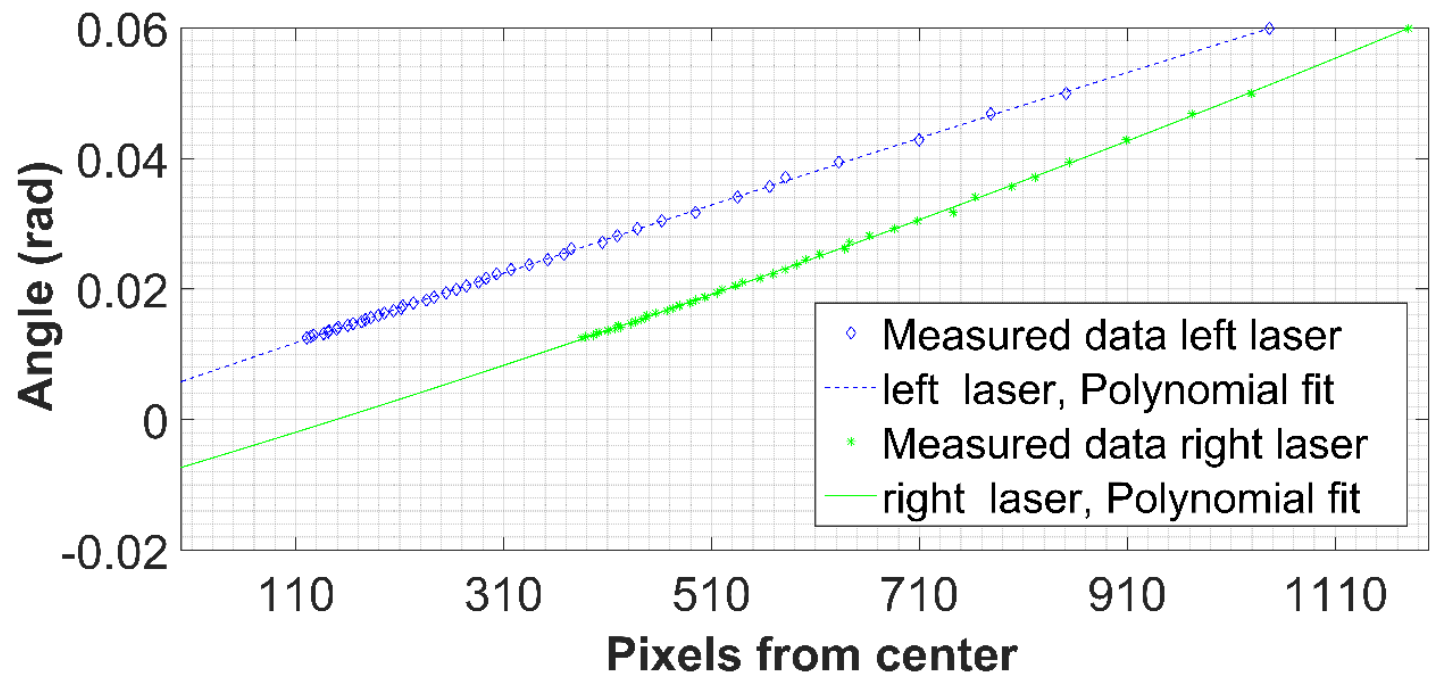

Fig. A.1. Relationship between the measuring angle $\theta^{\prime}$ and PFC.

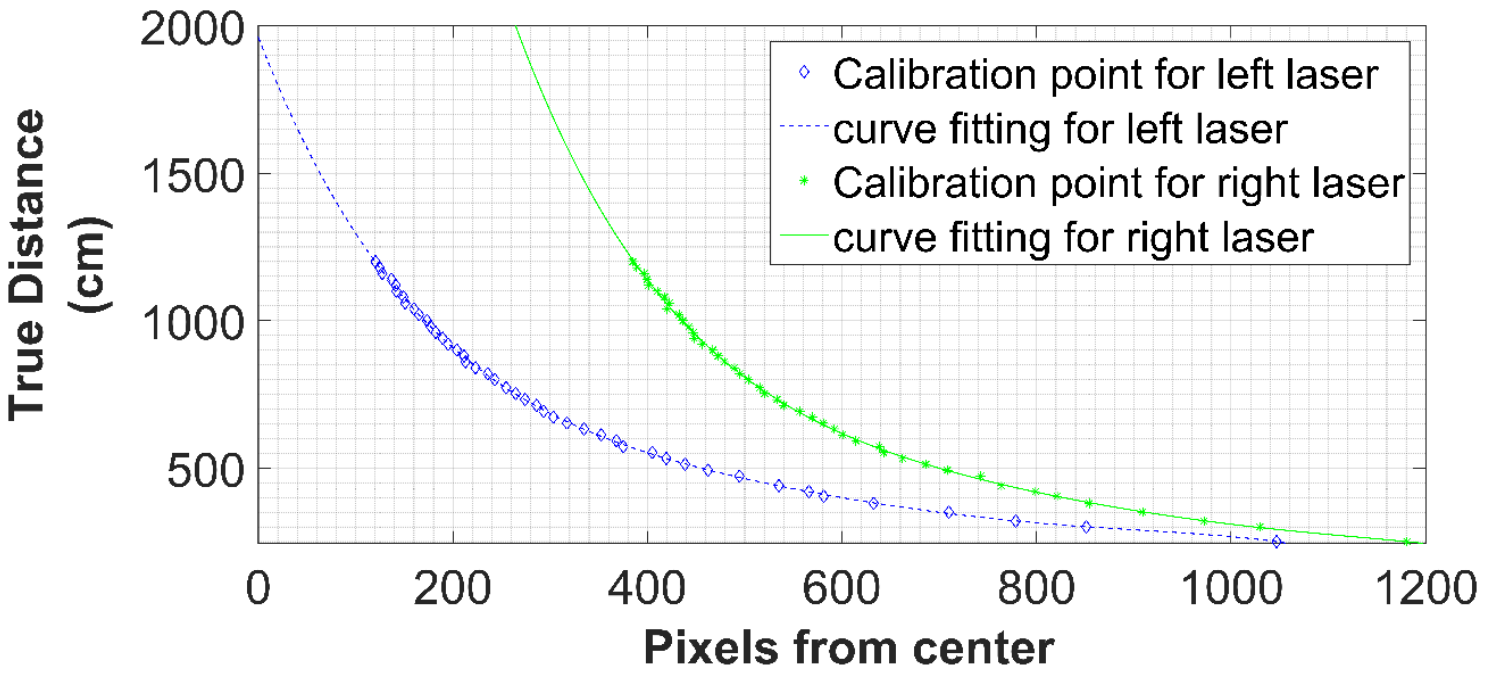

Fig. A.2. Relationship between the range to the target and PFC. 


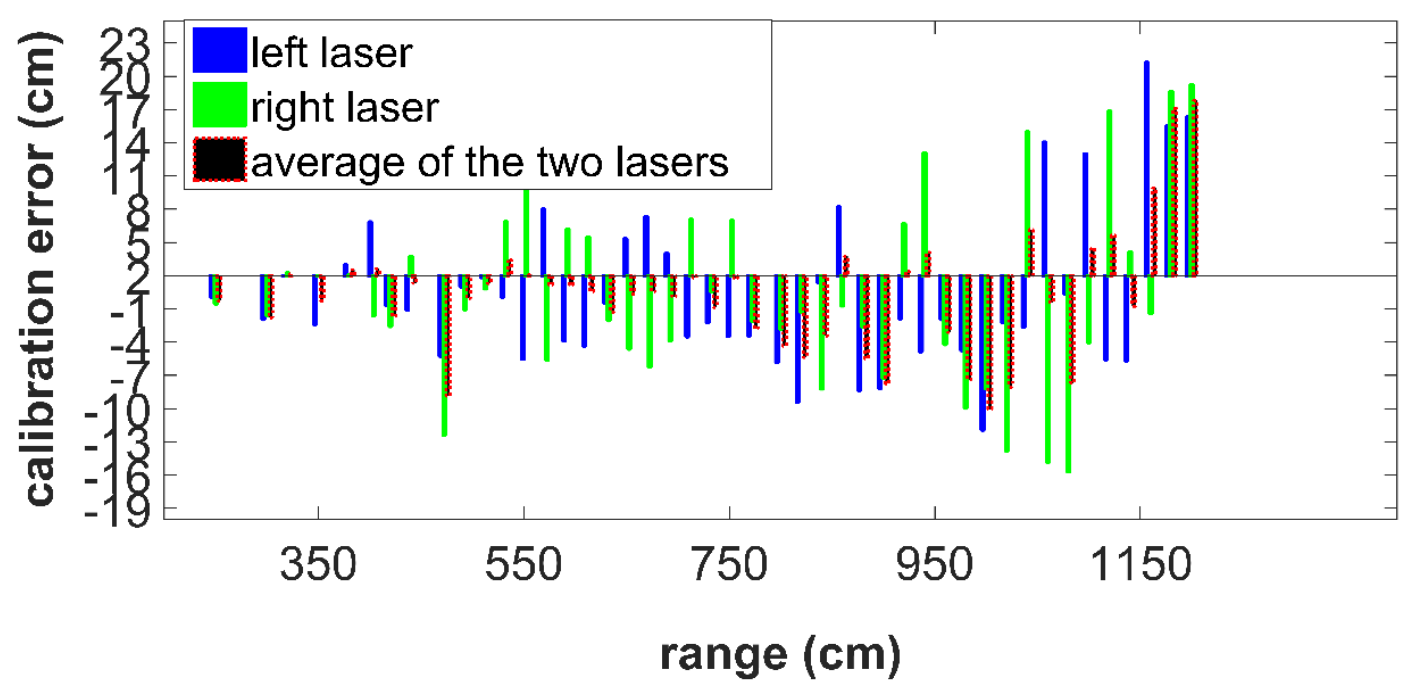

838

Fig. A.3. Calibration error for left laser (blue column), right laser (green column) and for the average (red column) between the two laser pointers. 
Fig. A.4. Airborne image of water surface taken by the CLDS. (a) the two laser dots are clearly identifiable (b) larger laser dots with contours that are less identifiable (c) multiple green laser dots caused by multiple reflection and scattering processes (d) laser light is reflected by the bottom (larger dots) and by the surface (smaller dots) 


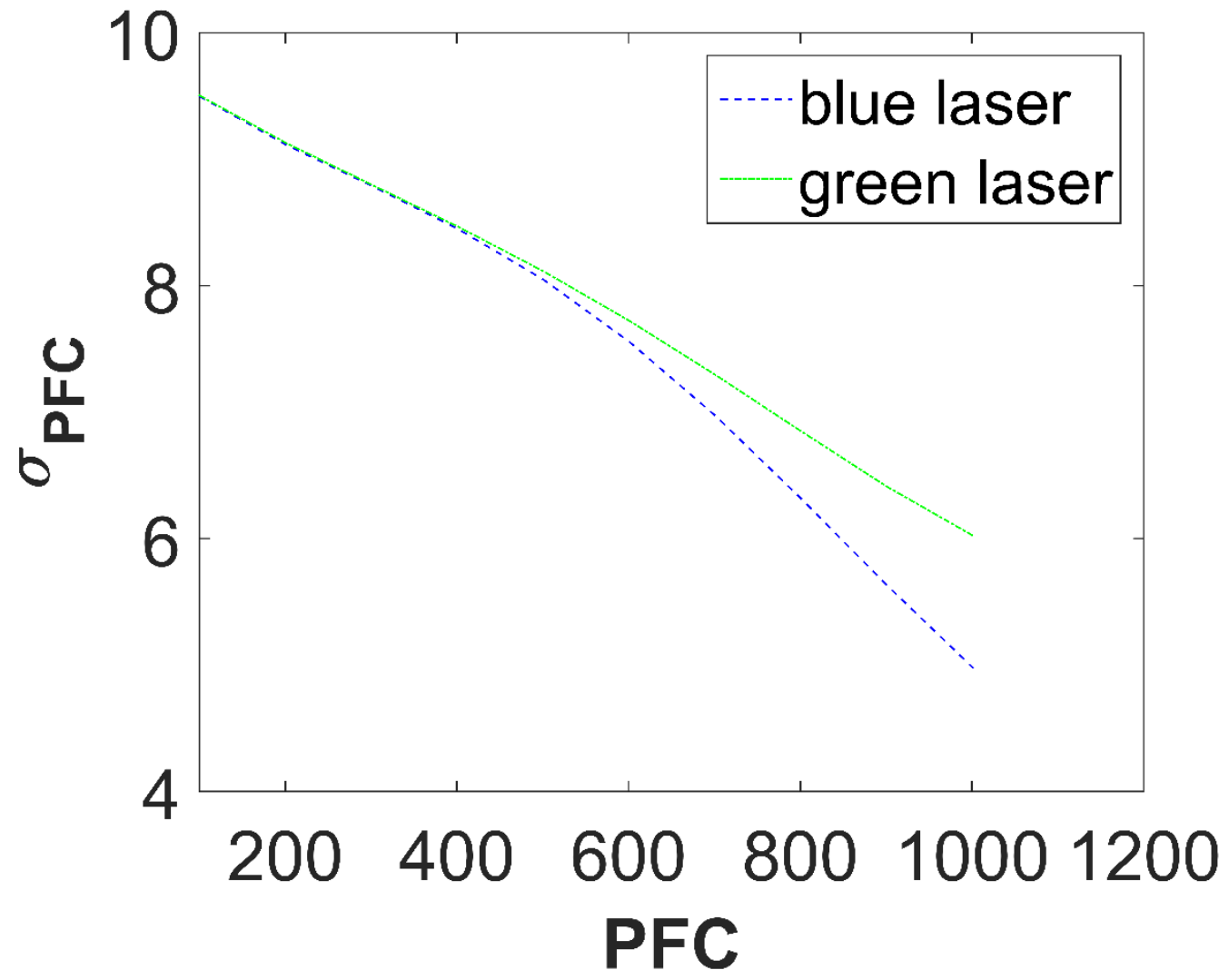

Fig. A.5. Uncertainty $\left(\sigma_{\mathrm{PFC}}\right)$ in computing the number of pixels as a function of PFC, for green and blue laser. 852 


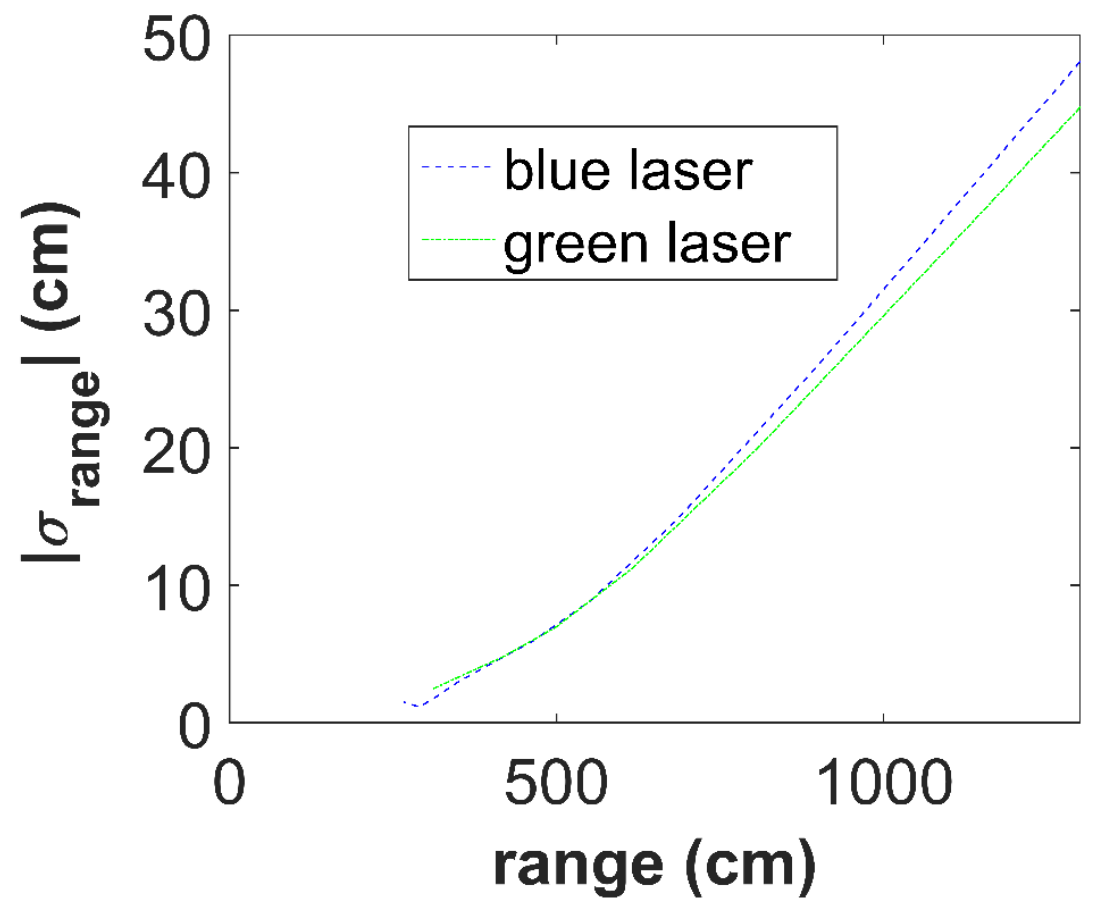

854

855

856

857

Fig. A.6. Absolute uncertainty in range measurement $\left(\left|\sigma_{\text {range }}\right|\right)$ as a function of the range to the target, for green and blue laser.

858

859

860

861

862 


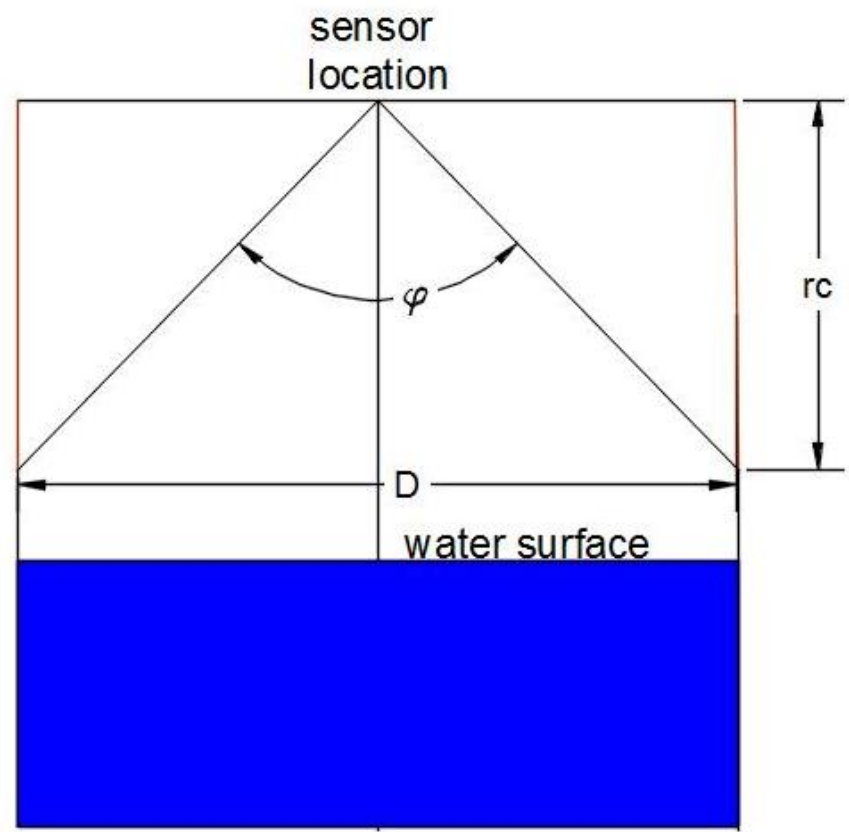

863

864

865 Fig. B.1. Schematic representation of the test conducted over the water well to retrieve beam divergence $(\varphi)$ for each of the sensors. $\mathrm{D}$ is the diameter of the water well, $\mathrm{r}_{\mathrm{c}}$ is the critical range.

\section{Tables}

Table 1. Technical performance of the sensors and of the GNSS receiver when tested in static mode.

\begin{tabular}{|c|c|c|c|c|}
\hline & $\begin{array}{l}\text { mean absolute } \\
\text { error (percentage } \\
\text { of the range) }\end{array}$ & $\begin{array}{l}\text { standard } \\
\text { deviation of the } \\
\text { stack }\end{array}$ & $\begin{array}{l}\text { Maximum ranging } \\
\text { distance }\end{array}$ & Beam divergence \\
\hline Radar & $-1.09 \%$ & $0.064 \mathrm{~m}$ & $\begin{array}{l}60 \mathrm{~m} \text { near field } \\
200 \mathrm{~m} \text { far field }\end{array}$ & $\sim 30^{\circ}$ \\
\hline Sonar & $0.98 \%$ & $0.007 \mathrm{~m}$ & $10 \mathrm{~m}$ & $>40^{\circ}$ \\
\hline CLDS & $1.5 \%$ & $2.3 \%$ of the range & $13 \mathrm{~m}$ & negligible \\
\hline GNSS receiver & negligible & Vertical & ------ & ----- \\
\hline
\end{tabular}


coordinates : 4-6

$\mathrm{cm}$ at 2 sigma

872

873

874

875 Table 2. Summary of the test flights over the lake. Each flight is named with the date (corresponding year is 2016). Ground truth

876 was measured with a RTK GNSS rover station connected to the network of reference stations. Statistics concern the water level

877 observations measured by subtracting the GNSS flight altitude from the range to water surface measured by each of the sensors.

878 Statistics are computed after removal of the observations that lie beyond $2 \sigma$.

Flight date $(\mathrm{dd} / \mathrm{mm} / \mathbf{2 0 1 6})$

Flight statistics

\begin{tabular}{|c|c|c|c|c|c|c|}
\hline & & $17 / 03$ & $04 / 04$ & 13/04 & $05 / 13$ & $27 / 05$ \\
\hline \multicolumn{2}{|l|}{ Ground truth (mamsl) } & missing & $\begin{array}{l}24.10 \pm 0.0 \\
6\end{array}$ & $\begin{array}{l}24.13 \pm 0.0 \\
6\end{array}$ & $\begin{array}{l}24.04 \pm 0.0 \\
6\end{array}$ & $\begin{array}{l}24.01 \pm 0.0 \\
6\end{array}$ \\
\hline \multirow{4}{*}{$\begin{array}{l}\text { Mean value (mamsl) of water } \\
\text { level retrieved by }\end{array}$} & radar & 24.10 & 24.11 & 24.20 & 24.11 & 24.02 \\
\hline & sonar & 23.50 & 23.93 & 20.01 & 27.05 & 38.45 \\
\hline & CLDS & missing & 24.29 & 24.81 & 24.82 & 23.93 \\
\hline & radar & 24.18 & 24.13 & 24.10 & 24.12 & 24.00 \\
\hline \multirow{3}{*}{$\begin{array}{l}\text { Mode value (mamsl) of water } \\
\text { level retrieved by }\end{array}$} & sonar & 24.40 & 24.14 & 24.08 & 24.65 & 27.50 \\
\hline & CLDS & missing & 21.27 & 24.56 & 24.41 & 20.66 \\
\hline & radar & 0.07 & 0.05 & 0.08 & 0.09 & 0.05 \\
\hline \multirow{2}{*}{$\begin{array}{l}\text { Standard Deviation }(\mathrm{m}) \text { in water } \\
\text { level retrieved by }\end{array}$} & sonar & 0.80 & 2.31 & 1.3 & 0.36 & 14.42 \\
\hline & CLDS & missing & 1.08 & 0.95 & 1.68 & 2.05 \\
\hline
\end{tabular}

879

880

881

882

883

884

885

886

887 
Table 3. Accuracy and ground footprint of different techniques for observing water level

\begin{tabular}{|c|c|c|c|c|}
\hline Location & Technique & Footprint & Accuracy & Reference \\
\hline Airborne & LIDARs & $20 \mathrm{~cm}-1 \mathrm{~m}$ & $4-22 \mathrm{~cm}$ & $\begin{array}{c}\text { (Hopkinson et al., } \\
\text { 2011) }\end{array}$ \\
\hline Spaceborne & $\begin{array}{c}\text { laser altimetry (e.g. } \\
\text { ICESat) }\end{array}$ & $50-90 \mathrm{~m}$ & $10 \mathrm{~cm}$ & (Phan et al., 2012) \\
\hline Spaceborne & $\begin{array}{l}\text { radar altimetry (e.g. } \\
\text { ERS2, Envisat, } \\
\text { Topex/Poseidon) }\end{array}$ & $400 \mathrm{~m}-2 \mathrm{~km}$ & $30-60 \mathrm{~cm}$ & (Frappart et al., 2006) \\
\hline Ground-based & $\begin{array}{c}\text { radar/sonar/pressure } \\
\text { transducers }\end{array}$ & negligible & $1 \mathrm{~mm}-10 \mathrm{~cm}$ & $\begin{array}{c}\text { Widely known } \\
\text { metrology }\end{array}$ \\
\hline UAV-borne & radar altimetry & negligible & $5-7 \mathrm{~cm}$ & $\begin{array}{c}\text { Methodology described } \\
\text { in this paper }\end{array}$ \\
\hline
\end{tabular}

893 Table B.1. Locations, settings and environmental conditions during static (on ground) tests. Coordinates are in WGS84. Country 894 is either Denmark (DK) or Italy $(I T)$. Range $(m)$ is the value measured by the water level dip meter. Water speed has qualitatively 895 been classified into no speed (still water), low (less than $0.4 \mathrm{~m} / \mathrm{s}$ ), medium (between 0.4 and $1 \mathrm{~m} / \mathrm{s}$ ), and high speed (more than 1 $896 \mathrm{~m} / \mathrm{s}$ ). Wind speed has been qualitatively classified into no wind, low (wind less than $2 \mathrm{~m} / \mathrm{s}$ ), medium (between $2 \mathrm{~m} / \mathrm{s}$ and $8 \mathrm{~m} / \mathrm{s}$ ), 897 and high wind speed (more than $8 \mathrm{~m} / \mathrm{s}$ ). Illumination has been qualitatively classified into artificial lightening, low (less than 20 898 000 lux), medium (between 20000 and 50000 lux), and high illumination (more than 50000 lux)

\begin{tabular}{|c|c|c|c|c|c|c|c|c|c|}
\hline Latitude & Longitude & River & $\begin{array}{l}\text { Cou } \\
\text { ntry }\end{array}$ & $\begin{array}{l}\text { Range } \\
\text { to water }\end{array}$ & $\begin{array}{l}\text { Date } \\
(\mathrm{dd} / \\
\mathrm{mm} / \\
\mathbf{2 0 1 5})\end{array}$ & $\begin{array}{l}\text { Time of } \\
\text { the day } \\
\text { (hh:mm) }\end{array}$ & $\begin{array}{l}\text { Flow } \\
\text { speed }\end{array}$ & Wind & Illumination \\
\hline 55.783431 & 12.515610 & Laboratory & DK & 0.63 & $2 / 11$ & $11: 20$ & no & no & artificial \\
\hline 55.775211 & 12.470266 & Mølleåen & DK & 1.60 & $20 / 11$ & $13: 30$ & low & medium & low \\
\hline 55.775211 & 12.470266 & Mølleåen & DK & 2.38 & $20 / 11$ & $11: 41$ & low & medium & low \\
\hline 55.775211 & 12.470266 & Mølleåen & DK & 2.58 & $20 / 11$ & $11: 52$ & low & medium & low \\
\hline 55.775211 & 12.470266 & Mølleåen & DK & 2.65 & $10 / 10$ & $11: 20$ & low & high & low \\
\hline 55.775211 & 12.470266 & Mølleåen & DK & 2.98 & $1 / 10$ & $14: 10$ & low & medium & low \\
\hline 55.775211 & 12.470266 & Mølleåen & DK & 3.10 & $10 / 10$ & $11: 25$ & low & high & low \\
\hline 55.775211 & 12.470266 & Mølleåen & DK & 3.49 & $10 / 10$ & $11: 49$ & low & high & low \\
\hline 44.909645 & 10.991254 & Sabbioncello & IT & 3.92 & $22 / 12$ & $16: 00$ & low & low & low \\
\hline 55.775211 & 12.470266 & Mølleåen & DK & 4.20 & $10 / 10$ & $14: 10$ & low & high & medium \\
\hline 55.775211 & 12.470266 & Mølleåen & DK & 4.35 & $1 / 10$ & $14: 33$ & low & medium & low \\
\hline 45.038994 & 10.965141 & $\begin{array}{l}\text { Canale } \\
\text { Bonifica }\end{array}$ & IT & 5.32 & $22 / 12$ & $13: 00$ & low & low & medium \\
\hline
\end{tabular}




\begin{tabular}{|c|c|c|c|c|c|c|c|c|c|}
\hline & & Parmigiana, & & & & & & & \\
\hline 45.029723 & 10.959166 & $\begin{array}{l}\text { Canale della } \\
\text { Bonifica } \\
\text { Reggiana } \\
\text { Montovana }\end{array}$ & IT & 7.10 & $22 / 12$ & $14: 05$ & low & low & low \\
\hline 45.029726 & 10.960432 & $\begin{array}{l}\text { Canale della } \\
\text { Bonifica } \\
\text { Parmigiana }\end{array}$ & IT & 7.33 & $22 / 12$ & $9: 30$ & low & low & low \\
\hline 44.650573 & 10.794755 & Secchia & IT & 9.79 & $29 / 10$ & $12: 00$ & medium & medium & medium \\
\hline 44.821261 & 10.994579 & Secchia & IT & 11.16 & $29 / 10$ & $12: 50$ & medium & medium & high \\
\hline 44.67578 & 10.860146 & Secchia & IT & 12.20 & $29 / 10$ & $13: 50$ & medium & medium & medium \\
\hline 45.008365 & 10.977453 & Secchia & IT & 12.72 & $29 / 10$ & $20: 30$ & medium & medium & low \\
\hline 44.727259 & 11.045292 & Panaro & IT & 12.97 & $29 / 10$ & $8: 30$ & medium & low & low \\
\hline
\end{tabular}

900 Table B.2. Summary of the test flights over the lake.

Flight date (dd/mm/2016)

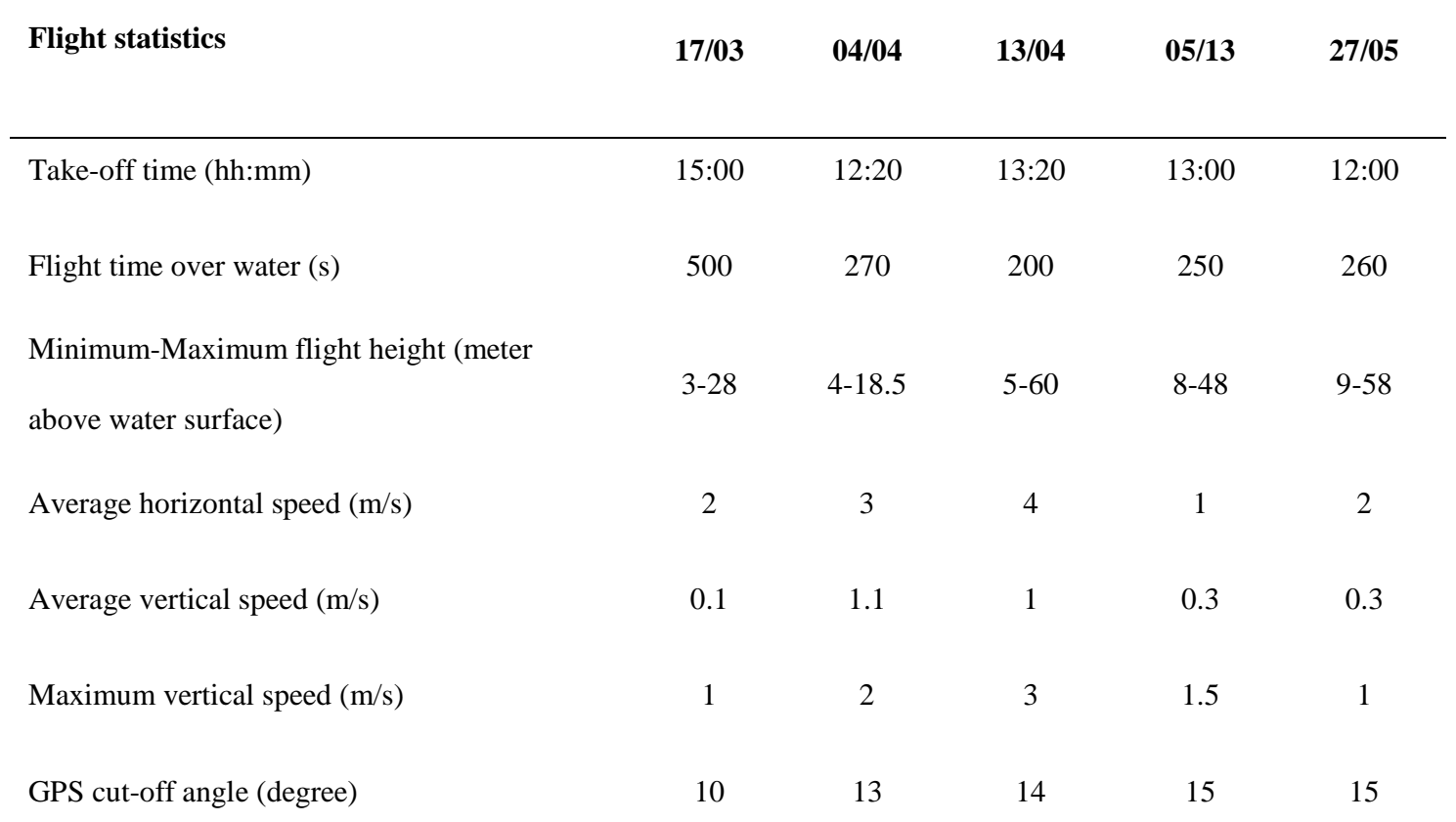

901

902

903 